TRANSACTIONS OF THE

AMERICAN MATHEMATICAL SOCIETY

Volume 352, Number 2, Pages 609-642

S 0002-9947(99)02277-1

Article electronically published on August 10, 1999

\title{
RARIFIED SUMS OF THE THUE-MORSE SEQUENCE
}

\author{
MICHAEL DRMOTA AND MARIUSZ SKAŁBA
}

\begin{abstract}
Let $q$ be an odd number and $S_{q, 0}(n)$ the difference between the number of $k<n, k \equiv 0 \bmod q$, with an even binary digit sum and the corresponding number of $k<n, k \equiv 0 \bmod q$, with an odd binary digit sum. A remarkable theorem of Newman says that $S_{3,0}(n)>0$ for all $n$. In this paper it is proved that the same assertion holds if $q$ is divisible by 3 or $q=4^{N}+1$. On the other hand, it is shown that the number of primes $q \leq x$ with this property is $o(x / \log x)$. Finally, analoga for "higher parities" are provided.
\end{abstract}

\section{INTRODUCTION}

The Thue-Morse sequence [9], [5] is defined by

$$
t_{n}=(-1)^{s(n)},
$$

where $s(n)$ denotes the number of ones in the binary representation of $n$. For any positive integer $q$ and $i \in \mathbf{Z}$ we denote

$$
S_{q, i}(n)=\sum_{0 \leq j<n, j \equiv i(\bmod q)} t_{j} .
$$

In 1969 Newman [10] proved a remarkable conjecture of L. Moser saying that for any $n \geq 1$

$$
S_{3,0}(n)>0 .
$$

More precisely, he proved that

$$
\frac{3^{\alpha}}{20}<\frac{S_{3,0}(n)}{n^{\alpha}}<5 \cdot 3^{\alpha} \quad \text { with } \alpha=\frac{\log 3}{\log 4} .
$$

In 1983 Coquet [1] provided an explicit precise formula for $S_{3,0}(n)$ by the use of a continuous function $\psi_{3}(x)$ with period 1 which is nowhere differentiable $\left(\eta_{3}(n) \in\right.$ $\{-1,0,1\})$ :

$$
S_{3,0}(n)=n^{\frac{\log 3}{\log 4}} \cdot \psi_{3}\left(\frac{\log n}{\log 4}\right)-\frac{\eta_{3}(n)}{3} .
$$

Furthermore, he was able to identify $\min \psi([0,1])>0$ and $\max \psi([0,1])$.

In general, (asymptotic) representations similar to (3) exist for any $S_{q, i}(n)$ (see [5] and section 2). But it is a non-trivial problem to decide whether the continuous function $\psi_{q, i}(x)$ has a zero or not. The only known examples where $\psi_{q}(x)=\psi_{q, 0}(x)$ has no zero are $q=3^{k} 5^{l}([6])$ and $q=17([7])$. (Note that the assertion that $\psi_{q, i}(x)$

Received by the editors July 6, 1995 and, in revised form, December 2, 1997.

1991 Mathematics Subject Classification. Primary 11B85; Secondary 11A63.

This work was supported by the Austrian Science Foundation, grant Nr. M 00233-MAT. 
has no zero is more or less equivalent to $S_{q, i}(n)>0$ for almost all $n$ or to $S_{q, i}(n)<0$ for almost all $n$; see section 2.)

Our first result provides infinitely many new examples where $\psi_{q}(x)$ has no zero.

Theorem 1. Suppose that $q$ is divisible by 3 or $q=4^{N}+1$. Then $S_{q, 0}(n)>0$ for almost all $n .^{1}$

However, if $q$ is prime then we can prove that there are only a few exceptions (e.g. Fermat primes). Let $\mathbf{P}_{t}, t \geq 1$, denote the set of those primes $p$ where the order $\operatorname{ord}_{p}(2)$ of 2 in the multiplicative group $(\mathbf{Z} / p \mathbf{Z})^{*}$ equals $\operatorname{ord}_{p}(2)=(p-1) / t$.

Theorem 2. There exists a constant $C>0$ such that for any $t \geq 1$ the primes $p \in \mathbf{P}_{t}$ satisfying $S_{p, 0}(n)>0$ for almost all $n$ are bounded by

$$
p \leq C t^{2} \log ^{2} t .
$$

Furthermore, the total number of primes $p \leq x$ with $S_{p, 0}(n)>0$ for almost all $n$ is $o(x / \log x)$ as $x \rightarrow \infty$.

The first part of Theorem 2 generalizes a result by the authors [2], where it is shown that 3 and 5 are the exceptional primes of $\mathbf{P}_{1}$ and 17 and possibly 41 those of $\mathbf{P}_{2}$. (In fact, $p=41$ is not exceptional, see section 3.)

It is surely a very difficult problem to decide whether there are infinitely many primes $p$ satisfying $S_{p, 0}(n)>0$ for almost all $n$ or not. Unfortunately our methods are not strong enough to settle this problem. But it should be noted that if there were only finitely many primes with this property, Theorem 1 would imply that there were only finitely many Fermat primes.

However, the methods to be developed are essentially sufficient to decide this problem for any concrete value $q$. For example, we can prove the following theorem.

Theorem 3. The only primes $p \leq 1000$ satisfying $S_{p, 0}(n)>0$ for almost all $n$ are $p=3,5,17,43,257,683$.

Note that $p=43 \in \mathbf{P}_{3}$ and $p=683 \in \mathbf{P}_{31}$ are not Fermat primes. ${ }^{2}$

We will prove Theorems 1 and 2 in sections 4 and 5 . The negative part of Theorem 3 is proved at the end of section 3 and the positive part at the end of section 4. Section 6 is devoted to the case of higher parities where similar phenomena appear. In section 2 we collect some basic facts on the fractal structure of $S_{q, i}(n)$, and in section 3 we discuss two different kinds of positivity phenomena.

\section{BASIC FACTS}

For any fixed positive integer $q$ and $i \in \mathbf{Z}$, set

$$
S_{q, i}(y, n)=\sum_{j<n, j \equiv i \bmod q} y^{s(j)},
$$

\footnotetext{
${ }^{1}$ The phrase "almost all" means "all but finitely many", i.e. there might be finitely many exceptions.

${ }^{2}$ Note that both 43 and 684 are of the form $\left(2^{2 N+1}+1\right) / 3$. Recently, by extending the methods of section 4, Leinfellner [8] showed that $q$ of the form $\left(2^{2 N+1}+1\right) / 3$ have the property that $S_{q, 0}(n)>0$ for almost all $n$.
} 
in which $n \geq 0$ and $y$ is a (complex) parameter. With help of these expressions we can determine the numbers

$$
\begin{aligned}
A_{q, i ; r, m}(n) & =|\{j<n: j \equiv i \bmod q, s(j) \equiv m \bmod r\}| \\
& =\frac{1}{r} \sum_{l=0}^{r-1} \zeta_{r}^{-m l} S_{q, i}\left(\zeta_{r}^{l}, n\right),
\end{aligned}
$$

where $r$ is a positive integer (which will be called a parity), $m \in \mathbf{Z}$, and $\zeta_{r}$ denotes the $r$-th primitive root of unity, $\zeta_{r}=\exp \left(\frac{2 \pi i}{r}\right)$.

Note that $S_{q, i}(y, n), 0 \leq i<q$, satisfies a simple generating relation if $n$ is a power of 2 :

$$
\sum_{i=0}^{q-1} S_{q, i}\left(y, 2^{k}\right) \zeta_{q}^{l i}=\prod_{j=0}^{k-1}\left(1+y \zeta_{q}^{l 2^{j}}\right),
$$

in which $\zeta_{q}=\exp \left(\frac{2 \pi i}{q}\right)$ denotes the $q$-th primitive root of unity and $l \in \mathbf{Z}$. Hence we directly obtain

$$
S_{q, i}\left(y, 2^{k}\right)=\frac{1}{q} \sum_{l=0}^{q-1} \zeta_{q}^{-l i} \prod_{j=0}^{k-1}\left(1+y \zeta_{q}^{l^{j}}\right) .
$$

Moreover, the obvious relation

$$
S_{q, i}\left(y, 2^{k}+n^{\prime}\right)=S_{q, i}\left(y, 2^{k}\right)+y S_{q, i-2^{k}}\left(y, n^{\prime}\right) \quad\left(n^{\prime}<2^{k}\right)
$$

can be used to calculate $S_{q, i}(n)$ inductively for any integer $n \geq 0$.

We will further need

$$
S(y, n)=\sum_{j<n} y^{s(j)}=\sum_{i=0}^{q-1} S_{q, i}(y, n)
$$

and the numbers

$$
\begin{aligned}
A_{r, m}(n) & =|\{j<n: s(j) \equiv m \bmod r\}| \\
& =\frac{1}{r} \sum_{l=0}^{r-1} \zeta_{r}^{-m l} S\left(\zeta_{r}^{l}, n\right) .
\end{aligned}
$$

$S\left(y, 2^{k}\right)$ is given by

$$
S\left(y, 2^{k}\right)=(1+y)^{k}
$$

and satisfies

$$
S\left(y, 2^{k}+n^{\prime}\right)=S\left(y, 2^{k}\right)+y S\left(y, n^{\prime}\right) \quad\left(n^{\prime}<2^{k}\right) .
$$

Our first aim is to describe the asymptotic behaviour of $A_{q, i ; r, m}(n)$. The natural leading term is $\frac{1}{q} A_{r, m}(n)$ :

$$
A_{q, i ; r, m}(n)=\frac{1}{q} A_{r, m}(n)+R_{q, i ; r, m}(n) .
$$

From (6), (8), (12), and (13) we obtain the representations

$$
A_{q, i ; r, m}\left(2^{k}\right)=\frac{1}{r q} \sum_{l_{1}=0}^{r-1} \zeta_{r}^{-l_{1} m} \sum_{l_{2}=0}^{q-1} \zeta_{q}^{-l_{2} i} \prod_{j=0}^{k-1}\left(1+\zeta_{r}^{l_{1}} \zeta_{q}^{l_{2} 2^{j}}\right)
$$


and

$$
A_{r, m}\left(2^{k}\right)=\frac{1}{r} \sum_{l_{1}=0}^{r-1} \zeta_{r}^{-l_{1} m}\left(1+\zeta_{r}^{l_{1}}\right)^{k}
$$

so that

$$
R_{q, i ; r, m}\left(2^{k}\right)=\frac{1}{r q} \sum_{l_{1}=0}^{r-1} \zeta_{r}^{-l_{1} m} \sum_{l_{2}=1}^{q-1} \zeta_{q}^{-l_{2} i} \prod_{j=0}^{k-1}\left(1+\zeta_{r}^{l_{1}} \zeta_{q}^{l_{2} 2^{j}}\right) .
$$

These Fourier expansions will be frequently used in the proofs of our main results.

From now on let $q$ be an odd positive integer and let $s=\operatorname{ord}_{q}(2)$ be the order of the multiplicative subgroup $\langle 2\rangle$ of $(\mathbf{Z} / q \mathbf{Z})^{*}$. (Since we are mainly interested in $A_{q, 0, r, m}(n)$, it is no real restriction to assume that $q$ is odd.) Furthermore, let

$$
\mathbf{S}_{q}(y, n)=\left(S_{q, 0}(y, n), \ldots, S_{q, q-1}(y, n)\right)^{t}
$$

denote the vector of $S_{q, i}(y, n)$. Let $\mathbf{e}_{0}, \ldots, \mathbf{e}_{q-1}$ denote the canonical basis of the $q$-dimensional vector space $\mathbf{C}^{q}$ and let $\mathbf{T}$ denote the matrix defined by $\mathbf{T} \mathbf{e}_{i}=\mathbf{e}_{i+1}$ $\left(\mathbf{e}_{q}=\mathbf{e}_{0}\right)$. The identity matrix is denoted by $\mathbf{I}$.

The following observations are more or less direct generalizations of [5].

Proposition 1. Let $\mathbf{M}(y)$ be defined by

$$
\mathbf{M}(y)=\prod_{m=0}^{s-1}\left(\mathbf{I}+y \mathbf{T}^{2^{m}}\right) .
$$

Then

$$
\mathbf{S}_{q}\left(y, 2^{s} n\right)=\mathbf{M}(y) \mathbf{S}_{q}(y, n) .
$$

Proof. By using the relations $s(2 j)=s(j)$ and $s(2 j+1)=s(j)+1$ we obtain

$$
\begin{aligned}
S_{q, i}(y, 2 n) & =\sum_{j<2 n, j \equiv i \bmod q} y^{s(j)} \\
& =\sum_{2 j<2 n, 2 j \equiv i \bmod q} y^{s(2 j)}+\sum_{2 j+1<2 n, 2 j+1 \equiv i \bmod q} y^{s(2 j+1)} \\
& =\sum_{j<n, j \equiv 2^{-1} i \bmod q} y^{s(j)}+y \sum_{j<n, j \equiv 2^{-1}(i-1) \bmod q} \\
& =S_{q, 2^{-1} i}(y, n)+y S_{q, 2^{-1}(i-1)}(y, n) .
\end{aligned}
$$

Hence, denoting by $\mathbf{U}$ the matrix defined by $\mathbf{U} \mathbf{e}_{i}=\mathbf{e}_{2 i}$, we have

$$
\mathbf{S}_{q}(y, 2 n)=(\mathbf{U}+y \mathbf{U T}) \mathbf{S}_{q}(y, n) .
$$

By using the property $\mathbf{U T}=\mathbf{T}^{2} \mathbf{U}$ it follows by induction that

$$
(\mathbf{U}+y \mathbf{U T})^{i}=\left(\prod_{m=1}^{i}\left(\mathbf{I}+y \mathbf{T}^{2^{m}}\right)\right) \mathbf{U}^{i} .
$$

Since $\mathbf{T}^{q}=\mathbf{U}^{s}=\mathbf{I}$, we directly obtain (19) by setting $i=s$. 
The eigenvalues of $\mathbf{T}$ are exactly the $q$-th roots of unity $\zeta_{q}^{l}, 0 \leq l<q$, with corresponding eigenvectors $\mathbf{v}_{l}=\sum_{i=0}^{q-1} \zeta_{q}^{-i l} \mathbf{e}_{i}$ which are orthogonal. Since $\mathbf{M}(y)$ is a polynomial in $\mathbf{T}$, the eigenvalues of $\mathbf{M}(y)$ are given by

$$
\lambda_{l}(y)=\prod_{m=0}^{s-1}\left(1+y \zeta_{q}^{l 2^{m}}\right)
$$

It is clear that $\lambda_{l}(y)=\lambda_{l^{\prime}}(y)$ if and only if $l^{\prime}\langle 2\rangle=l\langle 2\rangle$. (Observe that $\mathbf{l}=l\langle 2\rangle$ contains $\operatorname{ord}_{(q /(q, l))}(2)$ elements, where $(q, l)$ denotes the greatest common divisor of $q$ and $l$.) Appropriately we will write $\lambda_{\mathbf{l}}(y)$ instead of $\lambda_{l}(y)$ if $l \in \mathbf{l}$. Let $L$ denote the system of equivalence classes $\mathbf{l}=l\langle 2\rangle$. Then a basis of the eigenspace $V_{\mathbf{l}}$ corresponding to $\lambda_{\mathbf{l}}(y), \mathbf{l} \in L$, is given by $\mathbf{v}_{l}, l \in \mathbf{l}$. All these eigenspaces are orthogonal. $\mathbf{P}_{\mathbf{l}}, \mathbf{l} \in L$, will denote the orthogonal projection on $V_{\mathbf{l}}$. Furthermore, let $V^{(0)}$ denote the eigenspace corresponding to the eigenvalue 0 (if 0 is an eigenvalue), $V^{(s)}$ the subspace corresponding to eigenvalues of modulus $<1, V^{(1)}$ the subspace corresponding to those of modulus $1, V^{(u)}$ corresponding to those with modulus $>1$, and $V^{(m)}$ that corresponding to those eigenvalues with maximal modulus. Furthermore, let $\mathbf{P}^{(0)}, \mathbf{P}^{(s)}, \mathbf{P}^{(1)}, \mathbf{P}^{(u)}$, and $\mathbf{P}^{(m)}$ denote the orthogonal projections on $V^{(0)}, V^{(s)}, V^{(1)}, V^{(u)}$, and $V^{(m)}$, respectively.

Using these notations and the same methods as in [5], we immediately obtain a fractal representation for $\mathbf{S}_{q}(y, n)$.

Proposition 2. There exists a contiuous function $\mathbf{F}(y, \cdot): \mathbf{R}^{+} \rightarrow V^{(u)}$ satisfying

$$
\mathbf{F}\left(y, 2^{s} x\right)=\mathbf{M}(y) \mathbf{F}(y, x) \quad(x>0)
$$

and $\mathbf{P}_{u} \mathbf{S}_{q}(y, n)=\mathbf{F}(y, n)$. Consequently

$$
\mathbf{S}_{q}(y, n)=\mathbf{F}(y, n)+ \begin{cases}\mathcal{O}(1) & \text { if } V^{(1)}=\{0\} \\ \mathcal{O}(\log n) & \text { if } V^{(1)} \neq\{0\}\end{cases}
$$

Let $\left|\lambda_{\mathbf{l}}(y)\right|>1$. Then $\mathbf{G}_{\mathbf{l}}(y, t)=\lambda_{\mathbf{l}}(y)^{-t} \mathbf{P}_{\mathbf{l}} \mathbf{F}\left(y, 2^{s t}\right)$ is a continuous function $\mathbf{G}_{\mathbf{l}}(y, \cdot): \mathbf{R} \rightarrow V_{\mathbf{l}}$ which satisfies $\mathbf{G}_{\mathbf{l}}(y, t+1)=\mathbf{G}_{\mathbf{l}}(y, t)$. With $\alpha_{\mathbf{l}}(y)=$ $\left(\log \lambda_{\mathbf{l}}(y)\right) /(s \log 2)$ we finally obtain a fractal representation for $\mathbf{S}_{q}(y, n)$ :

$$
\mathbf{S}_{q}(y, n)=\sum_{\left|\lambda_{\mathbf{l}}(y)\right|>1} n^{\alpha_{\mathbf{l}}(y)} \mathbf{G}_{\mathbf{l}}\left(y, \frac{\log n}{s \log 2}\right)+\mathcal{O}(\log n) .
$$

We want to mention also that it is quite easy to evaluate $\mathbf{G}_{\mathbf{l}}(y, t)$ for special values of $t$ by using the representation (8):

$$
\begin{aligned}
S_{q, i}\left(y, 2^{k}\right) & =\frac{1}{q} \sum_{l=0}^{q-1} \zeta_{q}^{-l i} \lambda_{l}(y)^{a} \prod_{j=0}^{b-1}\left(1+y \zeta_{q}^{l 2^{j}}\right) \\
& =\frac{1}{q} \sum_{\mathbf{l} \in L}\left(2^{k}\right)^{\alpha_{1}(y)} \lambda_{\mathbf{l}}^{-b / s} \sum_{l \in \mathbf{l}} \zeta_{q}^{-i l} \prod_{j=0}^{b-1}\left(1+y \zeta_{q}^{l 2^{j}}\right),
\end{aligned}
$$

where $k=a s+b, 0 \leq b<s$. In particular, the first component of $\mathbf{G}_{\mathbf{l}}(y, 0)$ is non-zero.

Sometimes it would be more convenient to operate with real exponents instead of in general complex exponents $\alpha_{\mathbf{l}}(y)$. For example, if $\lambda_{\mathbf{l}}(y)^{r^{\prime}}$ is real and positive for 
some positive integer $r^{\prime}$, then we can use $\tilde{\mathbf{G}}_{\mathbf{l}}(y, t)=\lambda_{\mathbf{l}}(y)^{-r^{\prime} t} \mathbf{P}_{\mathbf{1}} \mathbf{F}\left(y, 2^{r^{\prime}}\right.$ st $)$ instead of $\mathbf{G}_{\mathbf{l}}(y, t)$ and $\tilde{\alpha}_{\mathbf{l}}(y)=\Re\left(\alpha_{\mathbf{l}}(y)\right)$ instead of $\alpha_{\mathbf{l}}(y)$. (Compare with [5].)

For the evaluation of $A_{q, i ; r, m}(n)$ we will need $\mathbf{S}_{q}\left(\zeta_{r}^{m}, n\right), 0 \leq m<r$. It is an easy exercise to show that $\arg \left(\lambda_{\mathbf{l}}\left(\zeta_{r}^{m}\right)\right)=s m \pi / r+m^{\prime} \pi$ for some $m^{\prime} \in \mathbf{Z}$. Thus $\lambda_{\mathbf{l}}\left(\zeta_{r}^{m}\right)^{r}$ is real and $\lambda_{\mathbf{l}}\left(\zeta_{r}^{m}\right)^{2 r}>0$. Hence it is always possible to operate with positive exponents.

Finally, observe that $S(y, n)$ can be treated in a similar fashion as above but much more easily. Using the relation $S(y, 2 n)=(1+y) S(y, n)$, it follows that there is a continuous function $F(y, x)$ satisfying $F(y, 2 x)=(1+y) F(y, x)$ in the case $|1+y|>1$ such that

$$
S(y, n)=F(y, n)=n^{\alpha} G\left(y, \frac{\log n}{\log 2}\right),
$$

where $\alpha(y)=\log (1+y) / \log 2$ and $G(y, t)=(1+y)^{-t} F\left(2^{t}\right)$. Furthermore, $S(y, n)$ $=\mathcal{O}(1)$ if $|1+y|<1$ and $S(y, n)=\mathcal{O}(\log n)$ if $|1+y|=1$.

Now the fractal representations for $A_{r, m}(n)$ and $R_{q, i ; r, m}(n)$ follow immediately.

Theorem 4. Let $q, r$ be positive integers such that $q$ is odd and $r \geq 2$. Set

$$
\begin{aligned}
\alpha_{r} & =\frac{\log \left(2 \cos \frac{\pi}{r}\right)}{\log 2} \quad(r>2), \\
\alpha_{q, r} & =\max _{0<m<r, 0<l<q} \frac{\log \left|\lambda_{l}\left(\zeta_{r}^{m}\right)\right|}{s \log 2} .
\end{aligned}
$$

Furthermore, let $r^{\prime}$ be the least positive integer such that $\lambda_{l}\left(\zeta_{r}^{m}\right)^{r^{\prime}}>0$ for those $\lambda_{l}\left(\zeta_{r}^{m}\right), 0<l<q, 0<m<r$, with largest modulus.

Then there exist real valued periodic continuous functions $\psi_{r, m}(x), \psi_{q, i ; r, m}(x)$, $0 \leq m<r, 0 \leq i<q$, with period 1 such that

$$
\begin{aligned}
A_{r, m}(n) & =\frac{n}{r}+ \begin{cases}(-1)^{m} \eta_{n} / 2 & (\text { if } r=2), \\
n^{\alpha_{r}} \cdot \psi_{r, m}\left(\frac{\log n}{2 r \log 2}\right)+\mathcal{O}\left(n^{\beta_{r}}\right) & (\text { if } r>2),\end{cases} \\
R_{q, i ; r, m}(n) & =n^{\alpha_{q, r}} \cdot \psi_{q, i ; r, m}\left(\frac{\log n}{2 r^{\prime} s \log 2}\right)+\mathcal{O}\left(n^{\beta_{q, r}}\right),
\end{aligned}
$$

where $\beta_{r}<\alpha_{r}, \beta_{q, r}<\alpha_{q, r}$, and $\eta_{n}=0$ if $n \equiv 0 \bmod 2$ and $\eta_{n}=t_{n}$ if $n \equiv 1 \bmod 2$.

Proof. Since $A_{r, m}(n)$ is given by (12) and $A_{q, i ; r, m}$ by (6) (compare also with (16) and (17)), it follows that the asymptotic leading term of $A_{r, m}(n)-n / r$ depends on the largest eigenvalue $\lambda_{0}\left(\zeta_{r}^{m}\right)=\left(1+\zeta_{r}^{m}\right)^{s}, 0<m<r$, and the asymptotic leading term of $R_{q, i ; r, m}(n)$ on the largest eigenvalue $\lambda_{l}\left(\zeta_{r}^{m}\right), 0<l<q, 0 \leq m<r$.

Since $\left|1+\zeta_{r}^{m}\right|=2\left|\cos \left(\frac{m \pi}{r}\right)\right|$ is maximal for $m=1$, we immediately obtain the asymptotic expansion for $A_{r, m}(n)$. (Note that $\beta_{r}=\log \left(2 \cos \frac{2 \pi}{r}\right) / \log 2$.)

Furthermore, since $\lambda_{l}(1)=1+\zeta_{q}^{l}+\zeta_{q}^{2 l}+\cdots+\zeta_{q}^{\left(2^{s}-1\right) l}=0$ for $0<l<q$, it is clear that $\alpha_{q, r}$ is the correct exponent in the asymptotic leading term of $R_{q, i ; r, m}(n)$.

Finally, $A_{2, m}(n)$ can be directly evaluated.

Remark. In this paper we will only discuss binary digits. But the above concept easily applies for arbitrary $b$-ary digit expansions. Let $s(j)$ be a sequence satisfying $s(b n+c)=s(n)+s(c)$ for $n \geq 0$ and $0 \leq c<b$. Let $\mathbf{S}_{q}(y, n)$ be defined as above 
and assume that $b$ and $q$ are relatively prime. Then

$$
\mathbf{S}_{q}(y, b n)=\mathbf{U}_{b}\left(\sum_{c=0}^{b-1} y^{s(c)} \mathbf{T}^{c}\right) \mathbf{S}_{q}(y, n),
$$

where $\mathbf{U}_{b} \mathbf{e}_{i}=\mathbf{e}_{b i}, 0 \leq i<q$, and $s=\operatorname{ord}_{q}(b)$. Hence $\mathbf{S}_{q}\left(y, b^{s} n\right)=\mathbf{M}_{b}(y) \mathbf{S}_{q}(y, n)$, where

$$
\mathbf{M}_{b}(y)=\prod_{m=0}^{b-1}\left(\sum_{c=0}^{b-1} y^{s(c)} \mathbf{T}^{c b^{m}}\right)
$$

and we are in the same position as above. All eigenvalues and eigenvectors of $\mathbf{M}_{b}(y)$ are known, and we immediately obtain a fractal representation for $\mathbf{S}_{q}(y, n)$. (In [5] only the case $b=r$ is mentioned.)

\section{Newman-Like Phenomena}

We want to discuss two kinds of positivity pheonmena:

$$
\begin{array}{ll}
A_{q, 0 ; r, 0}(n)>\max _{0<m<r} A_{q, 0 ; r, m}(n) \text { for almost all } n \geq 0, \\
R_{q, 0 ; r, 0}(n)>0 & \text { for almost all } \mathrm{n} \geq 0 .
\end{array}
$$

Newman's theorem $S_{3,0}(n)>0(n \geq 0)$ is precisely the same as

$$
A_{3,0 ; 2,0}(n)>A_{3,0 ; 2,1}(n) \text {. }
$$

Therefore (N1) is a natural generalization of this property. Recall that $R_{q, 0 ; r, m}(n)$ is the remainder term of $A_{q, 0 ; r, m}(n)$ if $\frac{1}{q} A_{r, m}(n)$ is considered as the "natural" leading term of $A_{q, 0 ; r, m}(n)$ (see section 2). Hence, (N2) means that the remainder term $R_{q, 0 ; r, 0}(n)$ is positive (for almost all $n$ ). We will now show that (N1) implies (N2) if $\alpha_{r} \neq \alpha_{q, r}$.

The following lemma provides a necessary condition for (N1).

Lemma 1. If (N1) holds then $\alpha_{r} \leq \alpha_{q, r}$.

Proof. Suppose that $\alpha_{r}>\alpha_{q, r}$. In this case (see Theorem 4) the asymptotic behaviour of $A_{q, 0 ; r, m}(n)$ is determined by $A_{r, m}(n)$. However, we will show that $A_{r, 0}\left(2^{(2 a+1) r}\right)<A_{r, m}\left(2^{(2 a+1) r}\right)$ for all $m \not \equiv 0 \bmod r$ and sufficiently large $a$. Therefore (N1) cannot occur.

Combining (13) and Theorem 4, we obtain

$$
A_{r, m}\left(2^{k}\right)-\frac{2^{k}}{r} \sim 2 \Re\left(\zeta_{r}^{-m}\left(1+\zeta_{r}\right)^{k}\right) .
$$

Since $\left(1+\zeta_{r}\right)^{r}$ is real and negative, everything follows.

Hence, if $\alpha_{r} \neq \alpha_{q, r}$ then (N1) implies

$$
R_{q, 0 ; r, 0}(n)>\max _{0<m<r} R_{q, 0 ; r, m}(n) \quad \text { for almost all } n \geq 0 .
$$

Finally, (23) always implies (N2). This follows from the following property.

\section{Lemma 2.}

$$
\sum_{m=0}^{r-1} R_{q, i ; r, m}(n)=\mathcal{O}(\log n)
$$

for all $i=0, \ldots, q-1$. 
Proof. From (17) we get

$$
\sum_{m=0}^{r-1} R_{q, i ; r, m}\left(2^{k}\right)=\frac{1}{q} \sum_{l=1}^{q-1} \zeta_{q}^{-l i} \prod_{j=0}^{k-1}\left(1+\zeta_{q}^{l 2^{j}}\right) .
$$

This means that the asymptotic behaviour of this sum is determined by the eigenvalues $\lambda_{l}(1)$, which are given by

$$
\lambda_{l}(1)=\prod_{j=0}^{s-1}\left(1+\zeta_{q}^{l 2^{j}}\right)=1 .
$$

Hence (24) follows.

Note that there are situations where (N2) holds although (N1) fails; see Theorem 8. However, in the "classical" case $r=2$ it is easy to verify that (N1) and (N2) are equivalent to $S_{q, 0}(-1, n)>0$ (for almost all $n$ ).

Before we prove further necessary conditions for (N1) and (N2), we want to mention that "converse" phenomena of the form $A_{q, 0 ; r, 0}(n)<\min _{0<m<r} A_{q, 0 ; r, m}(n)$ or $R_{q, 0 ; r, 0}(n)<0$ for almost all $n \geq 0$ do not exist.

Lemma 3. There exist infinitely many $n \geq 0$ such that

$$
A_{q, 0 ; r, 0}(n)>\max _{0<m<r} A_{q, 0 ; r, m}(n)
$$

and

$$
R_{q, 0 ; r, 0}(n)>0 .
$$

Proof. Let $s=\operatorname{ord}_{q}(2)$ and let $n=2^{2 r s \cdot a}$ for some $a \geq 0$. Then $\lambda_{\mathbf{l}}\left(\zeta_{r}^{l}\right)^{2 r a}>0$ for all $\mathbf{l} \in L$ and $l=0, \ldots, q-1$. Hence (25) and (26) follow from

$$
\begin{aligned}
& A_{q, 0 ; r, m}(n)=\frac{1}{q} A_{r, m}(n)+R_{q, 0 ; r, m}(n) \\
& =\frac{1}{r q} \sum_{l=0}^{r-1} \cos \left(2 \pi \frac{l m}{r}\right) \lambda_{0}\left(\zeta_{r}^{l}\right)^{2 r a}+\frac{1}{r q} \sum_{l_{1}=0}^{r-1} \cos \left(2 \pi \frac{l_{1} m}{r}\right) \sum_{\mathbf{0} \neq \mathbf{l} \in L}|\mathbf{l}| \lambda_{\mathbf{l}}\left(\zeta_{r}^{l_{1}}\right)^{2 r a} .
\end{aligned}
$$

Theorem 5. Let $q, r$ be positive integers such that $q$ is odd and $r \geq 2$. If $s=$ $\operatorname{ord}_{q}(2)$ and $r$ are coprime or if there exists an integer $r^{\prime}>0$ such that $\lambda_{l}\left(\zeta_{r}^{m}\right)^{r^{\prime}}<0$ for those $\lambda_{l}\left(\zeta_{r}^{m}\right), 0<l<q, 0<m<r$, with maximal modulus, then (N1) and (N2) fail.

Proof. We only prove that (N2) fails. Since $\lambda_{0}\left(\zeta_{r}\right)^{r}<0$, the following proof can be extended to contradict (N1).

Let $L_{m}$ denote the set of pairs $(\mathbf{l}, m), \mathbf{l} \in L, 0<m<r$, such that the eigenvalues $\lambda_{\mathbf{l}}\left(\zeta_{r}^{m}\right)$ have maximal modulus $\rho$. Then the asymptotic leading term of $R_{q, 0 ; m, 0}(n)$ only depends on these eigenvalues. In particular, we have

$$
R_{q, 0 ; m, 0}\left(2^{k s}\right) \sim \frac{1}{r q} \sum_{(\mathbf{1}, m) \in L_{m}}|\mathbf{l}| \lambda_{\mathbf{l}}\left(\zeta_{r}^{m}\right)^{k} .
$$

If there exists an integer $r^{\prime}>0$ such that $\lambda_{\mathbf{l}}\left(\zeta_{r}^{m}\right)^{r^{\prime}}<0$ for $(\mathbf{l}, m) \in L_{m}$, then $R_{q, 0 ; m, 0}\left(2^{a 2 r s+r^{\prime} s}\right)<0$ for all $a \geq 0$. 
Now suppose that $r$ and $s$ are coprime. Since $\arg \left(\lambda_{\mathbf{l}}\left(\zeta_{r}^{m}\right)\right)=m s \pi / r+\eta \pi$, where $\eta \in\{0,1\}$, any eigenvalue $\lambda_{\mathbf{l}}\left(\zeta_{r}^{m}\right)$ is not real. Set $\eta_{\mathbf{l}, m}=\lambda_{\mathbf{l}}\left(\zeta_{r}^{m}\right) / \rho$ for $(\mathbf{l}, m) \in L_{m}$. Then $\eta_{\mathbf{l}, m}$ are non-real $(2 r)$-th roots of unity. Thus

$$
\sum_{b=0}^{2 r-1} \sum_{(\mathbf{l}, m) \in L_{m}}|\mathbf{1}| \eta_{\mathbf{l}, m}^{b}=0
$$

and consequently there exists $b_{0}, 0<b_{0}<2 r$, such that

$$
\sum_{(\mathbf{1}, m) \in L_{m}}|\mathbf{l}| \lambda_{\mathbf{I}}\left(\zeta_{r}^{m}\right)^{b_{0}}=\rho^{b_{0}} \sum_{(\mathbf{1}, m) \in L_{m}}|\mathbf{l}| \Re\left(\eta_{\mathbf{1}, m}^{b}\right)<0 .
$$

Hence $R_{q, 0 ; m, 0}\left(2^{a 2 r s+b_{0} s}\right)<0$ for sufficiently large $a$.

With the help of Theorem 5 we will prove the negative part of Theorem 3 saying that primes $p \leq 1000, p \neq 3,5,17,43,257,683$, do not satisfy $S_{p, 0}(-1, n)>0$ for almost all $n$. First, we only have to consider $p \in \mathbf{P}_{t}$ with $t>2$. In [2] it is shown that $p=3$ and $p=5$ are the only exceptional primes in $\mathbf{P}_{1}$, and $p=17$ and possibly $p=41$ those of $\mathbf{P}_{2}$. (We will treat the case $p=41$ in a moment.) Next, it follows from Theorem 5 that we only have to pay attention to those primes $p \in \mathbf{P}_{t}, t>2$, with even $s=\operatorname{ord}_{p}(2)$, e.g. for $p=109 \in \mathbf{P}_{3}$ we have $s=36$. Finally, if there is $k<s$ with

$$
S_{p, 0}^{(m)}\left(-1,2^{k}\right)=\frac{1}{p} \sum_{j=0}^{s-1} \prod_{i=0}^{k-1}\left(1-\zeta_{p}^{l_{m} 2^{i+j}}\right)<0
$$

in which $\lambda_{m}=\lambda_{l_{m}}(-1)$ is the largest eigenvalue, then $S_{p, 0}\left(-1,2^{a s+k}\right)<0$ for sufficiently large $a$. For example, for $p=109$ we have $l_{m}=9$ and $S_{109,0}^{(m)}\left(-1,2^{6}\right)<0$. Hence, for $p=109$ there is no phenomenon of type (N1). Similarly it follows that $S_{41,0}^{(m)}\left(-1,2^{8}\right)<0$, and we really have to consider just primes $p \in \mathbf{P}_{t}$ with $t>2$.

Table 1 gives a list of all primes $p \leq 1000, p \in \mathbf{P}_{t}, t>2$, such that $s$ is even. Furthermore the largest eigenvalue $\lambda_{m}=\lambda_{l_{m}}(-1)$ is represented by $l_{m}$, and if there is $k<s$ such that $S_{p, 0}^{(m)}\left(-1,2^{k}\right)<0$ then $k$ is listed.

The only primes for which this method provides no answer are $p=43,257,683$. At the end of section 4 it will be shown that for these primes $S_{p, 0}(-1, n)>0$ for almost all $n$. This completes the proof of the negative part of Theorem 3 .

Remark. It is also an interesting problem to consider $A_{q, i ; r, m}(n)$ and $R_{q, i ; r, m}(n)$ $(0 \leq m<r)$ for some fixed $i \not \equiv 0 \bmod q$. For example, it is known that $A_{3,1 ; 2,0}(n)<$ $A_{3,1 ; 2,1}(n)$ for almost all $n \geq 0$ (see [3]). Most of our methods can be applied in these cases too. However, for the sake of shortness we restrict ourselves to the case $i=0$. 
TABLE 1

\begin{tabular}{|r|r|r|r|r||r|r|r|r|r|}
\hline$p$ & $s$ & $t$ & $l_{m}$ & $k$ & $p$ & $s$ & $t$ & $l_{m}$ & $k$ \\
\hline 43 & 14 & 3 & 7 & - & 499 & 166 & 3 & 11 & 12 \\
109 & 36 & 3 & 9 & 6 & 571 & 114 & 5 & 25 & 13 \\
113 & 28 & 4 & 5 & 13 & 577 & 144 & 4 & 13 & 15 \\
157 & 52 & 3 & 9 & 9 & 593 & 148 & 4 & 9 & 14 \\
229 & 76 & 3 & 3 & 8 & 617 & 154 & 4 & 17 & 13 \\
241 & 24 & 10 & 35 & 6 & 641 & 64 & 10 & 43 & 10 \\
251 & 50 & 5 & 17 & 8 & 643 & 214 & 3 & 11 & 6 \\
257 & 16 & 16 & 43 & - & 673 & 48 & 14 & 51 & 23 \\
277 & 92 & 3 & 3 & 19 & 683 & 22 & 31 & 113 & - \\
281 & 70 & 4 & 15 & 16 & 691 & 230 & 3 & 3 & 18 \\
283 & 94 & 3 & 3 & 9 & 733 & 244 & 3 & 9 & 11 \\
307 & 102 & 3 & 7 & 14 & 739 & 246 & 3 & 9 & 12 \\
331 & 30 & 11 & 25 & 13 & 811 & 270 & 3 & 5 & 38 \\
353 & 88 & 4 & 7 & 18 & 953 & 68 & 14 & 51 & 11 \\
397 & 44 & 9 & 23 & 17 & 971 & 194 & 5 & 25 & 10 \\
433 & 72 & 6 & 21 & 13 & 997 & 332 & 3 & 17 & 6 \\
457 & 76 & 6 & 31 & 20 & & & & & \\
\hline
\end{tabular}

\section{Proof of Theorem 1}

In the case of the usual parity $r=2$ we just have to discuss $S_{q, i}(-1, n)$ to obtain all informations needed. For short we will write $S_{q, i}(n), \lambda_{l}$, and $\mathbf{M}$ instead of $S_{q, i}(-1, n), \lambda_{l}(-1)$, and $\mathbf{M}(-1)$.

From an heuristic point of view integers of the form $q=4^{N}+1$ or $q=4^{N}-1$ are 'good candidates' for a phenomenon of type (N1). In both cases we have $s(j) \equiv 0 \bmod 2$ for $j \equiv 0 \bmod q, j<q 4^{N}+1$, i.e. $S_{q, 0}(n)$ is as positive as possible. (The first case is trivial. For the second case see Proposition 4.) In fact, Theorem 1 says that $S_{q, 0}(n)>0$ (for almost all $n$ ) for these $q$. However, an heuristic argument of this kind does not work in all cases. Suppose that $q=2^{2 N+1}-1$. Then $s(j) \equiv 1 \bmod 2$ for $j \equiv 0 \bmod q, j<q 2^{2 N+1}+1$, i.e. $S_{q, 0}(n)$ is as negative as possible. Furthermore, $s=\operatorname{ord}_{q}(2)=2 N+1$ is odd. Hence, by Theorem 5 $S_{q, 0}(n)<0$ for infinitely many $n$. But we know from Lemma 3 that we also have $S_{q, 0}(n)>0$ for infinitely many $n$.

Let $\mathbf{S}_{q}^{(m)}(n)=\left(S_{q, 0}^{(m)}(n), \ldots, S_{q, q-1}^{(m)}(n)\right)^{t}=\mathbf{P}^{(m)} \mathbf{S}_{q}(n)$. According to the above considerations it is sufficient to show that

$$
S_{q, 0}^{(m)}(n) \gg n^{\left(\log \lambda_{m}\right) /(s \log 2)},
$$

where $\lambda_{m}$ denotes the maximal eigenvalue, resp. $\min \psi_{q, 0 ; m, 0}>0$.

First we will discuss the case $3 \mid q$, where it is rather easy to identify $\lambda_{m}$.

Lemma 4. Suppose that $q$ is a positive odd integer. Then any eigenvalue

$$
\lambda_{l}=\prod_{m=0}^{s-1}\left(1-\zeta_{q}^{l 2^{m}}\right)
$$

of $\mathbf{M}$ is bounded by $\left|\lambda_{l}\right|<3^{s / 2}$ or $\lambda_{l}=3^{s / 2}$. 
The case $\lambda_{l}=3^{s / 2}$ appears if and only if $q \equiv 0 \bmod 3$ and $l \equiv q / 3 \bmod q$ or $l \equiv 2 q / 3 \bmod q$.

Proof. It is an elementary exercise to show that

$$
\left|1-z^{2}\right|<\sqrt{3} \text { and }\left|(1-z)\left(1-z^{2}\right)\right|<3
$$

if $|z|=1$ and $|1-z|>\sqrt{3}$. Furthermore $\left|1-z^{2}\right|=\sqrt{3}$ if $|z|=1$ and $|1-z|=\sqrt{3}$.

Now let $\lambda_{l}=\prod_{m=0}^{s-1}\left(1-\zeta_{q}^{l 2^{m}}\right)$ be an eigenvalue of $\mathbf{M}$. Let us consider a partition $M_{0}, M_{1}, M_{2}, M_{3}$ of the set $\{0,1, \ldots, s-1\}$, where $M_{0}$ consists of those $m$ with $\left|1-\zeta_{q}^{l 2^{m}}\right|=\sqrt{3}, M_{1}$ of those with $\left|1-\zeta_{q}^{l 2^{m}}\right|>\sqrt{3}$, and $M_{2}=M_{1}+1$. It is clear that either $M_{0}=\emptyset$ or $M_{0}=\{0,1, \ldots, s-1\}$. Furthermore $M_{1}, M_{2}, M_{3}$ are pairwise disjoint. If $M_{0}=\emptyset$ then

$$
\left|\lambda_{l}\right|=\prod_{m \in M_{1}}\left|\left(1-\zeta_{q}^{l 2^{m}}\right)\left(1-\zeta_{q}^{l 2^{m+1}}\right)\right| \prod_{m \in M_{3}}\left|1-\zeta_{q}^{l 2^{m}}\right|<3^{\left|M_{1}\right|} 3^{\left|M_{3}\right| / 2}=3^{s / 2} .
$$

On the other hand, if $M_{0}=\{0,1, \ldots, s-1\}$, then $s$ is even and $\lambda_{l}=3^{s / 2}$. Furthermore, the case $M_{0}=\{0,1, \ldots, s-1\}$ occurs only if $q \equiv 0 \bmod 3$ and $l \equiv q / 3 \bmod q$ or $l \equiv 2 q / 3 \bmod q$.

Lemma 5. Suppose that $q$ is an odd multiple of 3. Then

$$
\begin{aligned}
\left|S_{q, i}^{(m)}\left(2^{k}\right)\right| & \leq \frac{2}{q} 3^{k / 2} \quad(0 \leq i<q), \\
S_{q,-2^{j}}^{(m)}\left(2^{k}\right) & \leq 0 \quad(0 \leq j<s) \\
S_{q, 0}^{(m)}\left(2^{k}\right) & \geq \frac{\sqrt{3}}{q} 3^{k / 2} .
\end{aligned}
$$

Proof. Set $\omega=\zeta_{3}$. By (8) we have

$$
S_{q, i}^{(m)}\left(2^{k}\right)=\frac{1}{q}\left(\omega^{-i} \prod_{j=0}^{k-1}\left(1-\omega^{2^{j}}\right)+\omega^{i} \prod_{j=0}^{k-1}\left(1-\omega^{-2^{j}}\right)\right) .
$$

Since $\omega^{2^{j}}=\omega^{(-1)^{j}}$ and $\left|1-\omega^{ \pm 1}\right|=\sqrt{3}$, we immediately obtain the estimate (27). Furthermore,

$$
\prod_{j=0}^{k-1}\left(1-\omega^{2^{j}}\right)= \begin{cases}3^{k / 2} & \text { if } k \text { is even } \\ 3^{(k-1) / 2}(1-\omega) & \text { if } k \text { is odd. }\end{cases}
$$

Hence

$$
S_{q,-2^{i}}^{(m)}\left(2^{k}\right)= \begin{cases}-q^{-1} 3^{k / 2} & \text { if } k \text { is even, } \\ 0 & \text { if } k \text { is odd and } i \text { is even, } \\ -q^{-1} 3^{(k+1) / 2} & \text { if } k \text { and } i \text { are odd }\end{cases}
$$

and

$$
S_{q, 0}^{(m)}\left(2^{k}\right)= \begin{cases}2 q^{-1} 3^{k / 2} & \text { if } k \text { is even, } \\ q^{-1} 3^{(k+1) / 2} & \text { if } k \text { is odd, }\end{cases}
$$

which prove (28) and (29). 
Now suppose that $n=2^{k}+\delta 2^{k-1}+r$, where $\delta \in\{0,1\}$ and $r<2^{k-1}$. Then by using (9), (27), (28), and (29) we immediatly obtain

$$
\begin{aligned}
S_{q, 0}^{(m)}(n) & =S_{q, 0}^{(m)}\left(2^{k}\right)-\delta S_{q,-2^{k}}^{(m)}\left(2^{k-1}\right)+\sum_{j=0}^{k-2} \eta_{j} S_{q, i_{j}}^{(m)}\left(2^{j}\right) \\
& \geq\left(\frac{\sqrt{3}}{2}-\frac{\left(1-3^{-1 / 2}\right)^{-1 / 2}}{3}\right) \frac{2}{q} 3^{k / 2} \\
& >0.077 \cdot \frac{2}{q} 3^{k / 2} \gg n^{\left(\log \lambda_{m}\right) /(s \log 2)} .
\end{aligned}
$$

This proves Theorem 1 in the case $3 \mid q$.

The case $q=4^{N}+1$ is a little bit more involved. The first step is to identify the largest eigenvalue $\lambda_{m}$. Note that $s=4 N$.

Lemma 6. If $q=4^{N}+1$ then $\lambda_{m}$ is given by

$$
\lambda_{m}=\prod_{j=0}^{4 N-1}\left(1-\zeta_{q}^{l_{m} 2^{j}}\right)=c 3^{2 N}\left(1+\mathcal{O}\left(2^{-2 N}\right)\right),
$$

where $l_{m}=(q+1) / 3$ and $c=0.363247 \cdots>0$. Moreover, if $l \notin \mathbf{l}_{m}=l_{m}\langle 2\rangle$ then $\left|\lambda_{l}\right|<\lambda_{m}$.

Proof. First observe that for $0 \leq i<N$

$$
\begin{aligned}
\arg \zeta_{q}^{l_{m} 2^{2 i}} \in I_{1}=\left(\frac{2 \pi}{3}, \frac{5 \pi}{6}\right), & \arg \zeta_{q}^{l_{m} 2^{2 i+1}} \in I_{2}=\left(-\frac{2 \pi}{3},-\frac{\pi}{3}\right), \\
\arg \zeta_{q}^{l_{m} 2^{2 N+2 i}} \in I_{3}=\left(-\frac{5 \pi}{6},-\frac{2 \pi}{3}\right), & \arg \zeta_{q}^{l_{m} 2^{2 N+2 i+1}} \in I_{4}=\left(\frac{\pi}{3}, \frac{2 \pi}{3}\right) .
\end{aligned}
$$

This means that there are exactly $N$ elments $\zeta_{q}^{l_{m} 2^{i}}, 0 \leq i<4 N$, satisfying $\arg \zeta_{q}^{l_{m} 2^{i}} \in I_{1}$. Furthermore, the eigenvalue $\lambda_{m}$ is calculated by

$$
\begin{aligned}
\lambda_{m} & =\prod_{i=0}^{N-1}\left|1-\zeta_{q}^{l 4^{i}}\right|^{2}\left|1-\zeta_{q}^{2 l 4^{i}}\right|^{2} \\
& =\prod_{i=0}^{N-1} 16 \sin ^{2}\left(\frac{\pi}{3}+\frac{\pi 4^{i}}{3 q}\right) \sin ^{2}\left(\frac{2 \pi}{3}+\frac{2 \pi 4^{i}}{3 q}\right) \\
& =3^{2 N} \prod_{j=1}^{N} \frac{16}{9} \sin ^{2}\left(\frac{\pi}{3}+\frac{\pi}{34^{j}}\right) \sin ^{2}\left(\frac{2 \pi}{3}+\frac{2 \pi}{34^{j}}\right)\left(1+\mathcal{O}\left(2^{-2 N-j}\right)\right) \\
& =3^{2 N}\left(\prod_{j=1}^{\infty} \frac{16}{9} \sin ^{2}\left(\frac{\pi}{3}+\frac{\pi}{3 \cdot 4^{j}}\right) \sin ^{2}\left(\frac{2 \pi}{3}+\frac{2 \pi}{3 \cdot 4^{j}}\right)\right)\left(1+\mathcal{O}\left(2^{-2 N}\right)\right) \\
& =c 3^{2 N}\left(1+\mathcal{O}\left(2^{-2 N}\right)\right)
\end{aligned}
$$

If $\arg \zeta_{q}^{l} \in I_{1}$ for some $l \not \equiv 0 \bmod q$, then $\arg \zeta_{q}^{2 l} \in I_{2}, \arg \zeta_{q}^{2^{2 N} l} \in I_{3}$, and $\arg \zeta_{q}^{2^{2 N+1} l} \in I_{4}$. Hence, the number $N_{0}$ of elements $\zeta_{q}^{2^{i}}, 0 \leq i<4 N$, satisfying $\arg \zeta_{q}^{l 2^{i}} \in I_{1}$ is always bounded by $N_{0} \leq N$. 
The most interesting case appears if $N_{0}=N$. It is clear that this occurs if and only if $\arg \zeta_{q}^{l 2^{i}} \notin\left[-\frac{\pi}{3}, \frac{\pi}{3}\right]$ for all $i \geq 0$. Let us classify those $x \in(0,1)$ such that $z=e^{2 \pi(1+x) i / 3}$ satisfies $\arg z^{2^{i}} \notin\left[-\frac{\pi}{3}, \frac{\pi}{3}\right]$ for all $i \geq 0$.

Since $z \notin\left[-\frac{\pi}{3}, \frac{\pi}{3}\right]$ it follows that $z \notin\left[\frac{5 \pi}{6}, \frac{7 \pi}{6}\right]$, and consequently $z \notin\left[\frac{5 \pi}{12}, \frac{7 \pi}{12}\right] \cup$ $\left[-\frac{7 \pi}{12},-\frac{5 \pi}{12}\right]$ etc. By induction it follows that $\arg z$ must be contained in a zero set quite similar to the Cantor set. More precisely, the only possible values $x \in(0,1)$ are given by

$$
x=\sum_{n \geq 1} a_{n} 4^{-n}
$$

where $a_{n} \in\{0,3\}$ and there exist $n_{1}, n_{2} \geq 1$ with $a_{n_{1}}=0$ and $a_{n_{2}}=3$. If $z$ is in addition a $q$-th root of unity then $x$ must be of the form $x=k / q$, where $k \equiv 1 \bmod 3$ and $1 \leq k \leq 4^{N}$. Since

$$
\frac{1}{q}=\frac{4^{N}-1}{4^{2 N}-1}=\sum_{p \geq 0} \sum_{n=2 p N+N+1}^{2(p+1) N} 3 \cdot 4^{-n}
$$

we immediately obtain

$$
\frac{k}{q}=k \sum_{p \geq 1}\left(4^{N}-1\right) 4^{-2 p N}=\sum_{p \geq 1}\left((k-1) 4^{N}+\left(\left(4^{N}-1\right)-(k-1)\right)\right) 4^{-2 p N},
$$

and observe that the 4-adic digits $a_{n}$ of the digit expansion of $k / q, 1 \leq k \leq 4^{N}$, satisfy $a_{n} \in\{0,3\}$ for all $n \geq 1$ if and only if the 4-adic digit expansion of $k-1$ has the same property. (Evidently $k \equiv 1 \bmod 3$ in these cases.) This means that if we choose digits $b_{n} \in\{0,3\}, 1 \leq n \leq N$, and set

$$
k=1+\sum_{n=1}^{N} b_{n} 4^{N-n}
$$

then

$$
\frac{k}{q}=\sum_{p \geq 0}\left(\sum_{n=1}^{N} b_{n} 4^{-2 N p-n}+\sum_{n=1}^{N}\left(3-b_{n}\right) 4^{-2 N p-N-n}\right) .
$$

In this way we get all $q$-th roots of unity $z=\zeta_{q}^{l}$ with $\arg \zeta_{q}^{l} \in I_{1} \cup I_{3}$ such that $N_{0}=N$. Furthermore, the digits $b_{n}, 1 \leq n \leq N$, encode the distribution of $\zeta_{q}^{l 4^{i}}$. If $\zeta_{q}^{l}=e^{2 \pi\left(1+x_{0}\right) / 3}$ with $x_{0}=\sum_{n \geq 1} c_{n} 4^{-n}\left(c_{2 N p+n}=b_{n}, c_{2 N p+N+n}=3-b_{n}\right.$, $1 \leq n \leq N, p \geq 0)$, then $\zeta_{q}^{l 4^{i}}=e^{2 \pi\left(1+x_{i}\right) / 3}$, where $x_{i}=\sum_{n \geq 1} c_{n+i} 4^{-n}$. The periodicity $\zeta_{q}^{l 4^{2 N+i}}=\zeta_{q}^{l 4^{i}}$ is reflected by the periodic digit expansion of $x_{0}$. In particular, $\zeta_{q}^{l_{m}}$ corresponds to the digits $b_{n}=0,1 \leq n \leq N$. This means that $\zeta_{q}^{l_{m} 4^{i}}=e^{2 \pi\left(1+x_{i m}\right) / 3}$ are the only $q$-th roots of unity (with $\left.N_{0}=N\right)$, where one period of the digits of $x_{i m}$ contains just one subblock of the form 03. In other words, there is exactly one element $\zeta_{q}^{l_{m} 4^{i}}, 0 \leq i<N$, satisfying $\arg \zeta_{q}^{l_{m}} 4^{i} \in[19 \pi / 24,5 \pi / 6]$, namely $\zeta_{q}^{l_{m} 4^{N-1}}$. For any other $\zeta_{q}^{l}, l \notin \mathbf{l}_{m}$ (with $N_{0}=N$ ), there are at least two subblocks of the form 03 in any period of the digit expansion of $x_{0}$. Thus there exist $0 \leq i_{1}<i_{2}<N$ with $\arg \zeta_{q}^{l 4^{i_{1}}}, \arg \zeta_{q}^{l 4^{i_{2}}} \in[19 \pi / 24,5 \pi / 6]$. Consequently

$$
\lambda_{l}<3^{2 N} \frac{16^{2}}{9^{2}} \sin ^{4}\left(\frac{19 \pi}{24}\right) \sin ^{4}\left(\frac{19 \pi}{48}\right)=0.34899 \cdots 3^{2 N}<\lambda_{m} .
$$


The case $N_{0}<N$ is much easier. Let $J_{1}$ denote the set of $j, 0 \leq j<4 N$, such that $\arg \zeta_{q}^{l 2^{j}} \in I_{1}$. We assume that the elements $j_{i}, 0 \leq i<N_{0}$, of $J_{1}=$ $\left\{j_{0}, j_{1}, \ldots, j_{N_{0}-1}\right\}$ are 'ordered' in such a way that $\arg \zeta_{q}^{l 2^{j_{i}}} \leq \arg \zeta_{q}^{l 2^{j_{i+1}}}, 0 \leq i<$ $N_{0}-1$. (Recall that $\left|J_{1}\right|=N_{0}$.) Our first aim is to show that for any $i, 0 \leq i<N_{0}$, we have

$$
\arg \zeta_{q}^{l_{m} 4^{i}}<\arg \zeta_{q}^{l 2^{j_{i}}}
$$

Let $b_{i}, 1 \leq i<N$, denote the number of $j \in J_{1}$ satisfying $\arg \zeta_{q}^{l 2^{j}} \in I^{(i)}=$ $\left(\arg \zeta_{q}^{l_{m} 4^{i-1}}, \arg \zeta_{q}^{l_{m} 4^{i}}\right)$. Furthermore set $c_{i}=\sum_{1 \leq j \leq i} b_{j}$. Observe that

$$
c_{i} \leq i, \quad 1 \leq i<N
$$

immediately implies (30). Since $\arg \zeta_{q}^{l 2^{j}} \in I^{(i)}, 1 \leq i<N-1$, implies arg $\zeta_{q}^{l 2^{j+2}} \in$ $I^{(i+1)}$, we always have $b_{i+1} \geq b_{i}$. Set $a_{1}=b_{1}$ and $a_{i}=b_{i}-b_{i-1}, 2 \leq i<N$. Then $a_{i} \geq 0, b_{i}=\sum_{1 \leq j \leq i} a_{j}$, and $c_{i}=\sum_{1 \leq j \leq i}(i-j+1) a_{j}$.

Since $c_{N-1}=N_{0} \leq N-1$, condition (31) is satisfied for $i=N-1$. Now we show that $c_{i} \leq i$ implies $c_{i-1} \leq i-1$. Suppose that $c_{i-1} \geq i$; then we obtain $a_{1}+\cdots+a_{i}=c_{i}-c_{i-1} \leq 0$. Thus $a_{j}=0,1 \leq j \leq i$, which implies $c_{i-1}=0$ and contradicts $c_{i-1} \geq i$. This completes the proof of (31) and consequently that of $(30)$.

Let $J_{2}$ denote the set of $j, 0 \leq j<4 N$, such that $\arg \zeta_{q}^{l 2^{j}} \in(5 \pi / 6, \pi)$, and $J_{3}$ the set of those $j, 0 \leq j<4 N$, such that $\arg \zeta_{q}^{l 2^{j}} \in(0, \pi / 3)$. Clearly $N_{0}+\left|J_{2}\right|+\left|J_{3}\right|=N$ and

$$
\left|1-\zeta_{q}^{l 2^{j}}\right| \cdot\left|1-\zeta_{q}^{l 2^{j+1}}\right|<\left|1-\zeta_{q}^{l_{m} 2^{N-1}}\right| \cdot\left|1-\zeta_{q}^{l_{m} 2^{N}}\right|
$$

for $j \in J_{2} \cup J_{3}$. Therefore we can estimate $\lambda_{l}$ by

$$
\begin{aligned}
\lambda_{l}= & \prod_{j \in J_{1} \cup J_{2} \cup J_{3}}\left(\left|1-\zeta_{q}^{l 2^{j}}\right|^{2}\left|1-\zeta_{q}^{l 2^{j+1}}\right|^{2}\right) \\
= & \prod_{i=0}^{N_{0}-1}\left(16 \sin ^{2}\left(\frac{\arg \zeta_{q}^{l 2^{j_{i}}}}{2}\right) \sin ^{2}\left(\arg \zeta_{q}^{l 2^{j_{i}}}\right)\right) \\
& \cdot \prod_{j \in J_{2} \cup J_{3}}\left(16 \sin ^{2}\left(\frac{\arg \zeta_{q}^{l 2^{j}}}{2}\right) \sin ^{2}\left(\arg \zeta_{q}^{l 2^{j}}\right)\right) \\
< & \prod_{i=0}^{N_{0}-1}\left(16 \sin ^{2}\left(\frac{\arg \zeta_{q}^{l_{m} 2^{i}}}{2}\right) \sin ^{2}\left(\arg \zeta_{q}^{l_{m} 2^{i}}\right)\right) \\
& \cdot\left(16 \sin ^{2}\left(\frac{\arg \zeta_{q}^{l_{m} 2^{N-1}}}{2}\right) \sin ^{2}\left(\arg \zeta_{q}^{l_{m} 2^{N-1}}\right)\right) \\
< & \lambda_{m},
\end{aligned}
$$

which finishes the proof of Lemma 6 .

In order to complete the proof of Theorem 1 we need an analogon to Lemma 5. However, the situation is much more delicate. For the following estimates we use 
the notation

$$
c_{j}=\prod_{i>j}\left(\frac{2}{\sqrt{3}} \sin \left(\frac{\pi}{3}+(-1)^{i} \frac{\pi}{3} 2^{-i}\right)\right)=1+\mathcal{O}\left(2^{-j}\right) .
$$

The proof is completely elmentary and just uses the Fourier expansion (8) of $S_{q, i}\left(2^{k}\right)$, or its dominant term $S_{q, i}^{(m)}\left(2^{k}\right)$ corresponding to $\zeta_{q}^{l_{m}}$.

Lemma 7. Suppose that $q=4^{N}+1$ and $0 \leq k \leq 2 N$. Furthermore, let $i=0$ or $i=2^{k_{1}}+2^{k_{2}}+\cdots+2^{k_{l}}$, in which $k<k_{1}<k_{2}<\cdots<k_{l} \leq 2 N$, and set

$$
\begin{array}{rlrl}
w_{1} & =\sum_{l^{\prime}=1}^{l}(-1)^{k_{l^{\prime}}-k}, & w_{2} & =\sum_{l^{\prime}=1}^{l} 2^{k_{l^{\prime}}-k}, \\
w_{3}=\sum_{l^{\prime}=1}^{l}(-1)^{k_{l^{\prime}}-2 N}, & w_{4}=\sum_{l^{\prime}=1}^{l} 2^{k_{l^{\prime}}-2 N} .
\end{array}
$$

If $k \equiv 0 \bmod 2$, then

$$
\begin{aligned}
S_{q,-i}^{(m)}\left(2^{k}\right)=\frac{3^{k / 2}}{q}\left(2 \sum_{j=1}^{2 N-k} c_{j} \cos \left(\frac{\pi}{3} 2^{-j}+(-1)^{j} \frac{2 \pi}{3} w_{1}+\frac{2 \pi}{3} 2^{-j} w_{2}\right)\right. \\
+2 c_{0} \sum_{j=1}^{k} \frac{c_{j+2 N-k}}{c_{j}} \sin \left((-1)^{j} \frac{\pi}{6}+\frac{\pi}{3} 2^{-j}+\frac{\pi}{3} 2^{-j-2 N+k}\right. \\
\left.\left.+(-1)^{j} \frac{2 \pi}{3} w_{3}+\frac{2 \pi}{3} 2^{-j} w_{4}\right)+\mathcal{O}\left(2^{-k}\right)\right) \\
=\frac{3^{k / 2}}{q}\left(2(2 N-k) \cos \left(\frac{2 \pi}{3} w_{1}\right)+C_{1}\left(k ; k_{1}, \ldots, k_{l}\right)\right. \\
\left.+C_{2}\left(k ; k_{1}, \ldots, k_{l}\right)+\mathcal{O}\left(2^{-k}\right)+\mathcal{O}\left(2^{k-2 N}\right)\right)
\end{aligned}
$$

where the constants $C_{1}\left(k ; k_{1}, \ldots, k_{l}\right), C_{2}\left(k ; k_{1}, \ldots, k_{l}\right)$ are given by

$$
\begin{array}{r}
C_{1}\left(k ; k_{1}, \ldots, k_{l}\right) \\
=2 \sum_{j \geq 1}\left(c_{j} \cos \left(\frac{\pi}{3} 2^{-j}+(-1)^{j} \frac{2 \pi}{3} w_{1}+\frac{2 \pi}{3} 2^{-j} w_{2}\right)-\cos \left(\frac{2 \pi}{3} w_{1}\right)\right) \\
C_{2}\left(k ; k_{1}, \ldots, k_{l}\right) \\
=2 c_{0} \sum_{j \geq 1}\left(c_{j}^{-1} \sin \left((-1)^{j} \frac{\pi}{6}+\frac{\pi}{3} 2^{-j}+(-1)^{j} \frac{2 \pi}{3} w_{3}+\frac{2 \pi}{3} 2^{-j} w_{4}\right)\right. \\
\left.-\sin \left((-1)^{j} \frac{\pi}{6}+(-1)^{j} \frac{2 \pi}{3} w_{3}\right)\right),
\end{array}
$$

and $C_{2}\left(k ; k_{1}, \ldots, k_{l}\right)$ is uniformly bounded by $\left|C_{2}\left(k ; k_{1}, \ldots, k_{l}\right)\right| \leq 3.64$. 
If $k \equiv 1 \bmod 2$, then

$$
\begin{gathered}
S_{q,-i}^{(m)}\left(2^{k}\right)=\frac{3^{k / 2}}{q}\left(2 \sum_{j=1}^{2 N-k} c_{j} \cos \left((-1)^{j} \frac{\pi}{6}+\frac{\pi}{3} 2^{-j}+(-1)^{j} \frac{2 \pi}{3} w_{1}+\frac{2 \pi}{3} 2^{-j} w_{2}\right)\right. \\
+2 c_{0} \sum_{j=1}^{k} \frac{c_{j+2 N-k}}{c_{j}} \sin \left(\frac{\pi}{3} 2^{-j}+\frac{\pi}{3} 2^{-j-2 N+k}+(-1)^{j} \frac{2 \pi}{3} w_{3}+\frac{2 \pi}{3} 2^{-j} w_{4}\right) \\
\left.+\mathcal{O}\left(2^{-k}\right)\right) \\
=\frac{3^{k / 2}}{q}\left(2(2 N-k) \cos \left(\frac{\pi}{6}+\frac{2 \pi}{3} w_{3}\right)+D_{1}\left(k ; k_{1}, \ldots, k_{l}\right)\right. \\
\left.\quad+D_{2}\left(k ; k_{1}, \ldots, k_{l}\right)+\mathcal{O}\left(2^{-k}\right)+\mathcal{O}\left(2^{k-2 N}\right)\right),
\end{gathered}
$$

where the constants $D_{1}\left(k ; k_{1}, \ldots, k_{l}\right), D_{2}\left(k ; k_{1}, \ldots, k_{l}\right)$ are given by

$$
\begin{array}{r}
D_{1}\left(k ; k_{1}, \ldots, k_{l}\right)=2 \sum_{j \geq 0}\left(c_{j} \cos \left((-1)^{j} \frac{\pi}{6}+\frac{\pi}{3} 2^{-j}+(-1)^{j} \frac{2 \pi}{3} w_{1}+\frac{2 \pi}{3} 2^{-j} w_{2}\right)\right. \\
\left.-\cos \left(\frac{\pi}{6}+\frac{2 \pi}{3} w_{3}\right)\right) \\
D_{2}\left(k ; k_{1}, \ldots, k_{l}\right)=2 c_{0} \sum_{j \geq 1}\left(c_{j}^{-1} \sin \left(\frac{\pi}{3} 2^{-j}+(-1)^{j} \frac{2 \pi}{3} w_{3}+\frac{2 \pi}{3} 2^{-j} w_{4}\right)\right. \\
\left.-\sin \left((-1)^{j} \frac{2 \pi}{3} w_{3}\right)\right)
\end{array}
$$

and $D_{2}\left(k ; k_{1}, \ldots, k_{l}\right)$ is uniformly bounded by $\left|D_{2}\left(k ; k_{1}, \ldots, k_{l}\right)\right| \leq 2.22$.

Corollary 1. Suppose that $q=4^{N}+1$ and $0 \leq k \leq 2 N$. Then

$$
\begin{aligned}
& \left|S_{q,-i}^{(m)}\left(2^{k}\right)\right| \leq \frac{3^{k / 2}}{q}(2(2 N-k)+3.65), \quad\left(2^{k+1} \leq i<4^{N}+1\right), \\
& -S_{q,-2^{k+1}}^{(m)}\left(2^{k}\right) \geq \begin{cases}q^{-1} 3^{k / 2}((2 N-k)-2.674) & (k \equiv 0 \bmod 2), \\
q^{-1} 3^{k / 2} \cdot 1.453 & (k \equiv 1 \bmod 2),\end{cases} \\
& -S_{q,-2^{k+2}}^{(m)}\left(2^{k}\right) \geq \begin{cases}q^{-1} 3^{k / 2}((2 N-k)-0.669) & (k \equiv 0 \bmod 2), \\
q^{-1} 3^{k / 2}(\sqrt{3}(2 N-k)-5.12) & (k \equiv 1 \bmod 2),\end{cases} \\
& -S_{q,-2^{k+3}}^{(m)}\left(2^{k}\right) \geq \begin{cases}q^{-1} 3^{k / 2}((2 N-k)-2.358) & (k \equiv 0 \bmod 2), \\
q^{-1} 3^{k / 2} \cdot 4.791 & (k \equiv 1 \bmod 2),\end{cases} \\
& S_{q,-2^{k+1}-2^{k+2}}^{(m)}\left(2^{k}\right) \geq \begin{cases}q^{-1} 3^{k / 2}(2(2 N-k)-5.984) & (k \equiv 0 \bmod 2), \\
q^{-1} 3^{k / 2}(\sqrt{3}(2 N-k)-3.699) & (k \equiv 1 \bmod 2),\end{cases} \\
& S_{q, 0}^{(m)}\left(2^{k}\right) \geq \begin{cases}q^{-1} 3^{k / 2}(2(2 N-k)+0.831) & (k \equiv 0 \bmod 2), \\
q^{-1} 3^{k / 2}(\sqrt{3}(2 N-k)+1.262) & (k \equiv 1 \bmod 2),\end{cases}
\end{aligned}
$$

where all error terms $\mathcal{O}\left(2^{-2 N}\right)$ are neglected. 
Proof. (33) follows from Lemma 7 and the fact that

$$
\sum_{i=1}^{n} c_{i} \leq n+0.05 \quad(n \geq 1)
$$

The constants in (34)-(38) are easy to calculate.

Now, let $2^{4 N a} \leq n \leq 2^{4 N a+2 N}$ for some $a \geq 0$. Then the binary digit expansion of $n$ is given by

$$
n=d_{0} d_{1} \cdots d_{4 N a+k}=\sum_{j=0}^{2 n a+k} d_{j} 2^{4 N a+k-j},
$$

in which $d_{0}=1$ and $0 \leq k \leq 2 N$. Furthermore, let $d_{j_{i}}, 0 \leq i<s(n)$, denote exactly those digits with $d_{j_{i}}=1$. Then

$$
\begin{aligned}
& S_{q, 0}^{(m)}(n)=\sum_{i=0}^{s(n)-1}(-1)^{i} S_{q,-n_{i}}^{(m)}\left(2^{4 N a+k-j_{i}}\right) \\
& \quad=S_{q, 0}^{(m)}\left(2^{4 N a+k}\right)-S_{q,-2^{k}}^{(m)}\left(2^{4 N a+k-j_{1}}\right)+S_{q,-2^{k}-2^{k-j_{1}}}^{(m)}\left(2^{4 N a+k-j_{2}}\right) \mp \cdots,
\end{aligned}
$$

where

$$
n_{i}=\sum_{j<j_{i}} d_{j} 2^{4 N a+k-j} .
$$

Since $S_{q, i}^{(m)}\left(2^{4 N a+k}\right)=\lambda_{m}^{a} S_{q, i}^{(m)}\left(2^{k}\right)$, we can use Corollary 1 in order to estimate $S_{q, 0}^{(m)}(n)$ and $S_{q, 0}(n)$.

First, suppose that $k \equiv 0 \bmod 2$. In the case $d_{0}=1, d_{1}=d_{2}=d_{3}=0$ we have $j_{1} \geq 4$, and consequently

$$
\begin{aligned}
S_{q, 0}^{(m)}(n) & =S_{q, 0}^{(m)}\left(2^{4 N a+k}\right)+\sum_{i \geq 1}(-1)^{i} S_{q,-n_{j_{i}}}^{(m)}\left(2^{4 N a+k-j_{i}}\right) \\
& \geq \frac{\lambda_{m}^{a} 3^{k / 2}}{q}\left(2(2 N-k)+0.831-\sum_{i \geq 4}(2(2 N-k)+2 i+3.65) 3^{-i / 2}\right) \\
& \geq \frac{\lambda_{m}^{a} 3^{k / 2}}{q}(1.474(2 N-k)-2.951) .
\end{aligned}
$$

Hence, if $k \leq 2 N-3$ and $k \equiv 0 \bmod 2$ (i.e. $k \leq 2 N-4$ ), then $S_{q, 0}^{(m)}(n)>0$. If $d_{0}=1, d_{1}=d_{2}=0, d_{3}=1$, then we obtain in the same way

$$
\begin{aligned}
S_{q, 0}^{(m)}(n)= & S_{q, 0}^{(m)}\left(2^{4 N a+k}\right)-S_{q,-2^{k}}^{(m)}\left(2^{4 N a+k-3}\right)+\sum_{i \geq 2}(-1)^{i} S_{q,-n_{j_{i}}}^{(m)}\left(2^{4 N a+k-j_{i}}\right) \\
\geq & \frac{\lambda_{m}^{a} 3^{k / 2}}{q}\left(2(2 N-k)+0.831+3^{-3 / 2} 4.791\right. \\
& \left.-\sum_{i \geq 4}(2(2 N-k)+2 i+3.65) 3^{-i / 2}\right) \\
\geq & \frac{\lambda_{m}^{a} 3^{k / 2}}{q}(1.474(2 N-k)-2.029)
\end{aligned}
$$


Thus, $S_{q, 0}^{(m)}(n)>0$ if $k \leq 2 N-2$. Next, let $d_{0}=1, d_{1}=0, d_{2}=1$. Here we can verify that

$$
\begin{gathered}
2(2 N-k)+0.831+3^{-1}((2 N-k+2)-0.669) \\
-\sum_{i \geq 3}(2(2 N-k)+2 i+3.65) 3^{-i / 2} \\
=1.422(2 N-k)-4.363>0
\end{gathered}
$$

for $k \leq 2 N-4$. In the case $d_{0}=d_{1}=1, d_{2}=0$ we have

$$
\begin{aligned}
& 2(2 N-k)+0.831+3^{-1 / 2} \cdot 1.453-2(2 N-k) \cdot 0.456-5.638 \\
& \quad=1.088(2 N-k)-3.979>0
\end{aligned}
$$

if $k \leq 2 N-4$. Finally, if $d_{0}=d_{1}=d_{2}=1$ we can check that

$$
\begin{gathered}
2(2 N-k)+0.831+3^{-1 / 2} \cdot 1.453+3^{-1}(2(2 N-k+2)-5.984) \\
-2(2 N-k) \cdot 0.456-5.638=1.754(2 N-k)-4.578>0
\end{gathered}
$$

for $k \leq 2 N-3$.

Next, suppose that $k \equiv 1 \bmod 2$. If $d_{0}=1, d_{1}=d_{2}=d_{3}=0$, then

$$
\begin{aligned}
& \left.\sqrt{3}(2 N-k)+1.262-\sum_{i \geq 4}(2(2 N-k)+2 i+3.65) 3^{-i / 2}\right) \\
& \quad=1.206(2 N-k)-2.52>0
\end{aligned}
$$

for $k \leq 2 N-3$. If $d_{0}=1, d_{1}=d_{2}=0, d_{3}=1$, then

$$
\begin{gathered}
\sqrt{3}(2 N-k)+1.262+3^{-3 / 2}((2 N-k+3)-2.358) \\
-2(2 N-k) \cdot 0.263-3.782 \\
=1.786(2 N-k)-2.397>0
\end{gathered}
$$

for $k \leq 2 N-2$. If $d_{0}=1, d_{1}=0, d_{1}=1$, then

$$
\begin{gathered}
\sqrt{3}(2 N-k)+1.262+3^{-1}(\sqrt{3}(2 N-k+2)-5.12) \\
-2(2 N-k) \cdot 0.456-5.638 \\
=1.397(2 N-k)-4.928>0
\end{gathered}
$$

for $k \leq 2 N-4$. If $d_{0}=d_{1}=1, d_{2}=0$, then

$$
\begin{gathered}
\sqrt{3}(2 N-k)+1.262+3^{-1 / 2}((2 N-k+1)-2.674) \\
-2(2 N-k) \cdot 0.456-5.638 \\
=1.397(2 N-k)-5.343>0
\end{gathered}
$$

for $k \leq 2 N-4$. Finally, if $d_{0}=d_{1}=d_{2}=1$, then

$$
\begin{aligned}
& \sqrt{3}(2 N-k)+1.262+3^{-1 / 2}((2 N-k+1)-5.12) \\
& \quad+3^{-1}(\sqrt{3}(2 N-k+2)-3.699)-2(2 N-k) \cdot 0.456-5.638 \\
& \quad=1.974(2 N-k)-5.007>0
\end{aligned}
$$

for $k \leq 2 N-3$.

This implies $S_{q, 0}\left(2^{4 N a+k}+\cdots\right)>0$ if $k \leq 2 N-4$. The remaining cases $k=2 N$, $k=2 N-1, k=2 N-2$, and $k=2 N-3$ must be treated separately. 
First let $k=2 N$. By Lemma 7 it is easy to calculate $S_{q, 0}^{(m)}\left(2^{k}\right), S_{q,-2^{k}}^{(m)}\left(2^{k-1}\right)$, etc. up to an error term $\mathcal{O}\left(2^{-k}\right)=\mathcal{O}\left(2^{-2 N}\right)$. Let us consider a first example: $d_{0}=1$, $d_{1}=0, d_{2}=1, d_{3}=0, d_{4}=1$. We have

$$
\begin{aligned}
S_{q, 0}^{(m)}\left(2^{4 a N+2 N}\right) & =\lambda_{m}^{a} 3^{N}\left(2.20605 \cdots+\mathcal{O}\left(2^{-k}\right)\right), \\
S_{q,-2^{2 N}}^{(m)}\left(2^{4 a N+2 N-2}\right) & =\lambda_{m}^{a} 3^{N-1}\left(-4.4423 \cdots+\mathcal{O}\left(2^{-k}\right)\right), \\
S_{q,-2^{2 N}-2^{2 N-2}}^{(m)}\left(2^{4 a N+2 N-4}\right) & =\lambda_{m}^{a} 3^{N-2}\left(-0.1559 \cdots+\mathcal{O}\left(2^{-k}\right)\right),
\end{aligned}
$$

and

$$
\begin{aligned}
\sum_{i=3}^{s(n)-1}(-1)^{i} S_{q,-n_{i}}\left(2^{4 N a+2 N-j_{i}}\right) & \leq \lambda_{m}^{a} 3^{N} \sum_{i \geq 5}(2 i+3.56) 3^{-i / 2} \\
& \leq 2.4865 \lambda_{m}^{a} 3^{N}
\end{aligned}
$$

Hence

$$
\begin{aligned}
S_{q, 0}^{(m)}(n) & \geq 3^{2 N}\left(2.20605+3^{-1} 4.4423-3^{-2} 0.1559-2.4865+\mathcal{O}\left(2^{-2 N}\right)\right) \\
& >(3.6695-2.4865) 3^{2 N}
\end{aligned}
$$

which gives $S_{q, 0}\left(2^{4 a N}\left(2^{2 N}+2^{2 N-2}+2^{2 N-4}+\cdots\right)\right)>0$ for sufficiently large $a$.

All other cases can be treated in the same fashion. For completeness all relevant values are provided in Tables $2-5$. The first column corresponds to the leading digits $d_{0} d_{1} d_{2} \cdots d_{j}$ of $\left.n=2^{4 a N}\left(d_{0} 2^{k}+d_{1} 2^{k-1}+\cdots+d_{i} 2^{k-j}+\cdots\right)\right)$, the second one to the (approximate) value of the constant $c$ in

$$
S_{q, 0}^{(m)}\left(2^{4 a N}\left(d_{0} 2^{k}+d_{1} 2^{k-1}+\cdots+d_{j} 2^{k-j}\right)\right)=\lambda_{m}^{a} 3^{k / 2}\left(c+\mathcal{O}\left(2^{-k}\right)\right)
$$

and the third one to the error estimate

$$
d=\sum_{i \geq j+1}(2(2 N-k)+2 i+3.65) 3^{-i / 2} .
$$

For example, if $k=2 N$ and $d_{0} \cdots d_{j}=10101$, then $j=4, c=3.669508$ and $d=2.4865$.

Since $c>d$, in any case we have proved that $S_{q, 0}(n)>0$ for $2^{4 a N} \leq n \leq 2^{4 a N+2 N}$ if $a$ and $N$ are sufficiently large. The remaining cases $2^{4 a N+2 N}<n<2^{4(a+1) N}$ can be tackled in the same fashion. We just need to find an analoge to Lemma 7 and to consider several cases. Thus we have proved the second part of Theorem 1 for sufficiently large $N$. The above proof has neglected the error terms $\mathcal{O}\left(2^{-2 N}\right)$. It is an easy but messy job to take these errors into account. In fact, it turns out that the above proof gives the second part of Theorem 1 for $N \geq 5$. Therefore we just have to check the two cases $N=3$ and $N=4$. We omit the details, but it is clear how to proceed in these cases in order to prove that $S_{4^{N}+1,0}(n)>0$ for almost all $n$.

In the same fashion it is possible to prove $S_{43,0}(n)>0$ and $S_{683,0}(n)>0$ for almost all $n$. (Of course, a simple computer program assists us.) This completes the proof of Theorem 3 . 
TABLE $2 . k=N$

\begin{tabular}{|l|l|l|}
\hline$d_{0} \cdots d_{j}$ & $c$ & $d$ \\
\hline 100000 & 2.206052 & 1.611 \\
100001 & 2.820907 & 1.611 \\
10001 & 2.928609 & 2.4865 \\
10010 & 3.377196 & 2.4865 \\
10011 & 3.609011 & 2.4865 \\
10100 & 3.686833 & 2.4865 \\
10101 & 3.669508 & 2.4865 \\
10110 & 3.456902 & 2.4865 \\
10111 & 3.956975 & 2.4865 \\
11000 & 3.691269 & 2.4865 \\
11001 & 4.500393 & 2.4865 \\
1101 & 4.628219 & 3.781 \\
1110 & 4.908283 & 3.781 \\
1111 & 4.78918 & 3.781 \\
& \multicolumn{2}{|l}{} \\
\end{tabular}

TABLE $4 . k=N-2$

\begin{tabular}{|l|l|l|}
\hline$d_{0} \cdots d_{j}$ & $c$ & $d$ \\
\hline 1000 & 5.09167 & 4.832 \\
1001 & 6.11387 & 4.832 \\
1010 & 6.48795 & 4.832 \\
1011 & 6.12713 & 4.832 \\
1100 & 6.26171 & 4.832 \\
1101 & 7.11082 & 4.832 \\
1110 & 7.30221 & 4.832 \\
1111 & 7.18199 & 4.832 \\
\hline
\end{tabular}

TABLE $3 . k=N-1$

\begin{tabular}{|l|l|l|}
\hline$d_{0} \cdots d_{j}$ & $c$ & $d$ \\
\hline 10000 & 3.82099 & 2.79 \\
10001 & 3.96445 & 2.79 \\
10010 & 4.13854 & 2.79 \\
10011 & 4.36108 & 2.79 \\
10100 & 4.16599 & 2.79 \\
10101 & 3.72492 & 2.79 \\
10110 & 3.51240 & 2.79 \\
10111 & 3.75527 & 2.79 \\
11000 & 3.45378 & 2.79 \\
11001 & 4.26506 & 2.79 \\
11010 & 4.27048 & 2.79 \\
11011 & 4.79882 & 2.79 \\
11100 & 4.88619 & 2.79 \\
11101 & 4.67129 & 2.79 \\
11110 & 4.07916 & 2.79 \\
11111 & 4.55637 & 2.79 \\
\hline
\end{tabular}

TABLE $5 . k=N-3$

\begin{tabular}{|l|l|l|}
\hline$d_{0} \cdots d_{j}$ & $c$ & $d$ \\
\hline 1000 & 6.65934 & 5.3581 \\
1001 & 7.36031 & 5.3581 \\
1010 & 7.89254 & 5.3581 \\
1011 & 7.13277 & 5.3581 \\
1100 & 7.42538 & 5.3581 \\
1101 & 8.63985 & 5.3581 \\
1110 & 9.29522 & 5.3581 \\
1111 & 8.79174 & 5.3581 \\
\hline
\end{tabular}

\section{Proof of Theorem 2}

The crucial step of the proof of Theorem 2 is contained in the following lemma.

Lemma 8. Let $p$ be an odd prime number and $s=\operatorname{ord}_{p}(2)$. Then

$$
S_{p, 0}\left(2^{4 k s-2}\right)=\frac{1}{p} \sum_{\mathbf{1} \in L} \lambda_{\mathbf{l}}^{4 k}\left(\frac{s}{2}-\frac{1}{4} \sum_{l \in \mathbf{l}} \frac{1}{1-\Re \zeta_{p}^{l}}\right) .
$$

Proof. Since $\lambda_{\mathbf{l}}^{4}$ is real for all eigenvalues $\lambda_{\mathbf{l}}=\prod_{l \in \mathbf{l}}\left(1-\zeta_{p}^{l}\right)$ and since

$$
S_{p, 0}\left(2^{4 k s-2}\right)=\frac{1}{p} \sum_{\mathbf{1} \in L} \lambda_{\mathbf{l}}^{4 k} \sum_{l \in \mathbf{l}} \frac{1}{\left(1-\zeta_{p}^{l}\right)\left(1-\zeta_{p}^{2 l}\right)},
$$

(39) follows from

$$
\Re\left(\frac{1}{(1-z)\left(1-z^{2}\right)}\right)=\frac{1}{2}-\frac{1}{4(1-\Re z)},
$$

in which $z \in \mathbf{C}$ has modulus $|z|=1$. 
The next lemma ensures that

$$
\frac{s}{2}<\frac{1}{4} \sum_{l \in \mathrm{l}} \frac{1}{1-\Re \zeta_{p}^{l}}
$$

for all $\mathbf{l} \in L$ if $p \in \mathbf{P}_{t}$ is sufficiently large. Hence $S_{p, 0}\left(2^{4 k s-2}\right)<0$ for all $k \geq 1$.

Lemma 9. Suppose that $p \in \mathbf{P}_{t}$ and that $p \geq(2 t \log p)^{2}$. Then

$$
\sum_{l \in \mathbf{l}} \frac{1}{1-\Re \zeta_{p}^{l}}>\frac{1}{8 \pi^{2}} \frac{p^{3 / 2}}{t^{2} \log p}
$$

Proof. By assumption $p \geq 2 t p^{1 / 2} \log p$. Hence by the Polya-Vinogradov inequality [12, p. 86 , Aufgabe 12 b]

$$
\left|\left\{k \in \mathbf{l}: 0<k \leq 2 t p^{1 / 2} \log p\right\}\right|>p^{1 / 2} \log p
$$

for all $\mathbf{l} \in L \backslash\{0\}$. Consequently

$$
\begin{aligned}
\sum_{l \in \mathbf{l}} \frac{1}{1-\Re \zeta_{p}^{l}} & =\sum_{l \in \mathbf{l}} \frac{1}{2 \sin ^{2}\left(\frac{l \pi}{p}\right)}>\frac{p^{2}}{2 \pi^{2}} \sum_{l \in \mathbf{l}} \frac{1}{l^{2}} \\
& >\frac{p^{2}}{2 \pi^{2}} \frac{p^{1 / 2} \log p}{\left(2 t p^{1 / 2} \log p\right)^{2}}=\frac{1}{8 \pi^{2}} \frac{p^{3 / 2}}{t^{2} \log p} .
\end{aligned}
$$

Now the first part of Theorem 2 follows from the next proposition.

Proposition 3. Suppose that $p \in \mathbf{P}_{t}$ satisfies $S_{p, 0}(n)>0$ for almost all $n$. Then

$$
p^{1 / 2} \leq 16 \pi^{2} t \log p,
$$

i.e., if $S_{p, 0}(n)>0$ for almost all $n$, then $s=\operatorname{ord}_{p}(2) \leq 16 \pi^{2} p^{1 / 2} \log p$.

Proof. It is clear that we just have to consider primes $p$ with $p^{1 / 2} \geq 2 t \log p$. If $p^{1 / 2}>16 \pi^{2} t \log p$, then Lemma 9 would imply

$$
\frac{s}{2}-\frac{1}{4} \sum_{l \in \mathbf{l}} \frac{1}{1-\Re \zeta_{p}^{l}}<\frac{p}{2 t}-\frac{1}{32 \pi^{2}} \frac{p^{3 / 2}}{t^{2} \log p}<0,
$$

and by using Lemma 8 we would obtain $S_{p, 0}\left(2^{4 k s-2}\right)<0$ for all $k \geq 1$.

In order to finish the proof of Theorem 2 we just have to mention a result by Erdős [4] saying that for any sequence $\varepsilon_{p} \rightarrow 0($ as $p \rightarrow \infty)$

$$
\left|\left\{p \leq x: s=\operatorname{ord}_{p}(2)<p^{1 / 2+\varepsilon_{p}}\right\}\right|=o\left(\frac{x}{\log x}\right) .
$$

Remark. Theorem 2 also says that the number $A_{t}$ of primes $p \in \mathbf{P}_{t}$ satisfying $S_{p, 0}(n)>0$ for almost all $n$ is bounded by $A_{t} \leq C p^{2} \log ^{2} p$. However, this bound can be essentially sharpened. A theorem of Titchmarsh [11, p. 147] says that for all $a, 0<a<1$, there exists a constant $C=C(a)$ such that

$$
\pi(x ; k, l)<C \frac{x}{\varphi(k) \log x}
$$

for all $1 \leq k \leq x^{a}$ and $0 \leq l<k$ with $\operatorname{gcd}(l, k)=1$. Since $p \in \mathbf{P}_{t}$ satisfies $p \equiv 1 \bmod t$, we get

$$
A_{t}=\mathcal{O}\left(t^{2}(\log t) / \varphi(t)\right)
$$


Furthermore, $\varphi(t)>c t /(\log \log t)$ for some constant $c>0$ (see [11, p. 24]). Hence

$$
A_{t}=\mathcal{O}(t \log t \log \log t) .
$$

Comparing the above properties with Theorem 4, we find that the fractal function $\psi_{p}(x)=\psi_{p, 0}(x)$ has a zero near $x=1$. It is also an interesting problem to determine other zeroes and sign changes of $\psi_{p}(x)$. In [2] it is shown that for almost all primes $p \in \mathbf{P}_{1}$ the fractal function $\psi_{p}(x)$ has a zero near $x=1 / 2$. Furthermore, a similar result may be expected for $\mathbf{P}_{2}$. If $|\Im(L(2, \chi))|>40 \pi^{2} p^{-3 / 2}$, where $\chi$ denotes the biquadratic character $\bmod p \in \mathbf{P}_{2}$, then $\psi_{p}(x)$ has a zero near $x=1 / 2$. Hence there is a connection between zeroes of $\psi_{p}(x)$ and properties of Dirichlet $L$ series. In what follows we will extend this connection to arbitrary $t$. However, we are unable to prove the properties of $L$-series. Nevertheless by numerical evidence (see [2]) the zeroes of $\psi_{p}$ seem to be very well dispersed. Therefore we conjecture that the $L$-series in question satisfy the proposed properties (43) and (44).

Let $p \in \mathbf{P}_{t}$, and denote by $\lambda_{m}$ the eigenvalue of largest modulus. If $s=\operatorname{ord}_{p}(2)$ is odd, then all eigenvalues $\lambda_{l}$ are imaginary and $r^{\prime}=4$, which means that $\psi_{p}\left(\frac{1}{2}\right)<0$ corresponds to $S_{p, 0}^{(m)}\left(2^{(4 a+2) s}\right)<0$. Hence the same arguments as in the proof of Theorem 2 give

$$
S_{p, 0}^{(m)}\left(2^{(4 a+2) s-2}\right)>0
$$

providing a sign change of $\psi_{p}(x)$ near $x=\frac{1}{2}$ for sufficiently large $p$. If $s=\operatorname{ord}_{p}(2)$ is even, then $2^{s / 2} \equiv-1 \bmod p$, and consequently all eigenvalues $\lambda_{l}$ are real and positive. Hence $\lambda_{m}>0$ and $r^{\prime}=1$. Let $\lambda_{m}=\prod_{i=0}^{s-1}\left(1-\zeta_{p}^{l_{m} 2^{i}}\right)$ and set

$$
a_{j}=\prod_{i=0}^{s / 2-1}\left(1-\zeta_{p}^{l_{m} 2^{j+i}}\right) .
$$

Then

$$
\begin{aligned}
S_{p, 0}^{(m)}\left(2^{a s+s / 2}\right) & =\frac{\lambda_{m}^{a}}{p} \sum_{j=0}^{s-1} a_{j}, \\
S_{p, 0}^{(m)}\left(2^{a s+s / 2-1}\right) & =\frac{\lambda_{m}^{a}}{p} \sum_{j=0}^{s-1} \frac{a_{j}}{1-\zeta_{p}^{l_{m} 2^{j}}}, \\
S_{p, 0}^{(m)}\left(2^{a s+s / 2-2}\right) & =\frac{\lambda_{m}^{a}}{p} \sum_{j=0}^{s-1} \frac{a_{j}}{\left(1-\zeta_{p}^{l_{m} 2^{j}}\right)\left(1-\zeta_{p}^{l_{m} 2^{j+1}}\right)} .
\end{aligned}
$$

Since $2^{s / 2} \equiv-1 \bmod p$ it follows that $\zeta_{p}^{l_{m} 2^{s / 2}}=\zeta_{p}^{-l_{m}}$. Hence $a_{j+1}=-a_{j} \zeta_{p}^{-l_{m} 2^{j}}$ and

$$
\begin{aligned}
\sum_{j=0}^{s-1} a_{j} & =a_{0} \zeta_{p}^{l_{m}} \sum_{j=0}^{s-1}(-1)^{j} \zeta_{p}^{-l_{m} 2^{j}} \\
\sum_{j=0}^{s-1} \frac{a_{j}}{1-\zeta_{p}^{l_{m} 2^{j}}} & =a_{0} \zeta_{p}^{l_{m}} \sum_{j=0}^{s-1}(-1)^{j} \frac{\zeta_{p}^{-l_{m} 2^{j}}}{1-\zeta_{p}^{l_{m} 2^{j}}}, \\
\sum_{j=0}^{s-1} \frac{a_{j}}{\left(1-\zeta_{p}^{l_{m} 2^{j}}\right)\left(1-\zeta_{p}^{l_{m} 2^{j+1}}\right)} & =a_{0} \zeta_{p}^{l_{m}} \sum_{j=0}^{s-1}(-1)^{j} \frac{\zeta_{p}^{-l_{m} 2^{j}}}{\left(1-\zeta_{p}^{l_{m} 2^{j}}\right)\left(1-\zeta_{p}^{l_{m} 2^{j+1}}\right)} .
\end{aligned}
$$


First, suppose that $s \equiv 2 \bmod 4$, i.e. $s / 2$ is odd. Then $\overline{a_{0}}=a_{s / 2}=(-1)^{s / 2} a_{0} \zeta_{p}^{2 l_{m}}$ implies that $a_{0} \zeta_{p}^{l_{m}}$ is imaginary. Since

$$
\frac{1}{z(1-z)}=\frac{1}{z}+\frac{1}{1-z}
$$

and

$$
\Im\left(\frac{1}{1-z}\right)=\frac{\Im z}{2(1-\Re z)}
$$

for $|z|=1$, we directly get

$$
\sum_{j=0}^{s-1}(-1)^{j} \frac{\zeta_{p}^{-l_{m} 2^{j}}}{1-\zeta_{p}^{l_{m} 2^{j}}}=\sum_{j=0}^{s-1}(-1)^{j} \zeta_{p}^{-l_{m} 2^{j}}+\frac{1}{2} \sum_{j=0}^{s-1}(-1)^{j} \frac{i \Im \zeta_{p}^{l_{m} 2^{j}}}{1-\Re \zeta_{p}^{l_{m} 2^{j}}} .
$$

Let $b$ be a generator of $G=(\mathbf{Z} / p \mathbf{Z})^{*} /\langle 4\rangle$, i.e. all residue classes $\bmod p$ are parametrized by $b^{i} 4^{j}, 0 \leq i \leq 2 t-1,0 \leq j \leq s / 2-1$, and $\chi_{k}, 1 \leq k \leq 2 t$, Dirichlet characters defined by $\chi_{k}\left(b^{i} 4^{j}\right)=\zeta_{2 t}^{i k}$. (Obviously the $\chi_{k}, 1 \leq k \leq 2 t$, constitute the character group of $G$.) If

$$
g_{\chi_{k}}=\sum_{n=0}^{p-1} \chi_{k}(n) \zeta_{p}^{n}
$$

denote the corresponding Gauss sums

$$
S_{1}=\sum_{j=0}^{s-1}(-1)^{j} \zeta_{p}^{-l_{m} 2^{j}}=\frac{1}{2 t} \sum_{k=1}^{2 t} \zeta_{2 t}^{k i_{m}}\left(1-(-1)^{k}\right) g_{\chi_{k}},
$$

in which $b^{i_{m}} \equiv l_{m}$ mod $p$. Furthermore, its absolute value can be estimated by $\left|S_{1}\right| \leq \sqrt{p}$. Now set

$$
h_{\chi_{k}}=\sum_{n=0}^{p-1} \chi_{k}(n) \frac{\Im \zeta_{p}^{n}}{1-\Re \zeta_{p}^{n}}=\sum_{n=0}^{p-1} \chi_{k}(n) \cot \frac{n \pi}{p}=\frac{p}{\pi}\left(1-(-1)^{k}\right) L\left(1, \chi_{k}\right) .
$$

Then

$$
S_{2}=\frac{1}{2} \sum_{j=0}^{s-1}(-1)^{j} \frac{i \Im \zeta_{p}^{l_{m} 2^{j}}}{1-\Re \zeta_{p}^{l_{m} 2^{j}}}=i \frac{p}{2 \pi t} \sum_{k=1}^{2 t} \zeta_{2 t}^{k i_{m}}\left(1-(-1)^{k}\right)^{2} L\left(1, \chi_{k}\right) .
$$

Note that $S_{1}$ and $S_{2}$ are imaginary. This representation is interesting if $\left|S_{2}\right|>\sqrt{p}$. If $\operatorname{sgn}\left(i S_{1}\right) \neq \operatorname{sgn}\left(i S_{2}\right)$, then it is clear that there is a sign change of $\psi_{p}(x)$ near $x=\frac{1}{2}$. If $\operatorname{sgn}\left(i S_{1}\right)=\operatorname{sgn}\left(i S_{2}\right)$, then it is an easy exercise to show that $S_{p, 0}^{(m)}\left(2^{a s+s / 2}\right)$ and $S_{p, 0}^{(m)}\left(2^{a s}\left(2^{s / 2}+2^{s / 2-1}\right)\right.$ have different signs. Therefore, if $p \in \mathbf{P}_{t}, s \equiv 2 \bmod 4$, and

$$
\left|\frac{\sqrt{p}}{4 \pi t} \sum_{k=1}^{2 t} \zeta_{2 t}^{k i_{m}}\left(1-(-1)^{k}\right)^{2} L\left(1, \chi_{k}\right)\right|>1,
$$

then there is a sign change of $\psi_{p}(x)$ near $x=\frac{1}{2}$. For example, if $p \in \mathbf{P}_{1}$ and $p>163$, then Dirichlet's class number formula and the fact that the class number $h$ of the corresponding quadratic field satisfies $h>1$ show that this case appears (see $[2])$. 
Finally, suppose that $p \in \mathbf{P}_{t}$ and that $s / 2$ is even, i.e. $s \equiv 0 \bmod 4$. Here $a_{0} \zeta_{p}^{l_{m}}$ is real and consequently $S_{1}$ is real, too. Furthermore, $\Re(1 /(1-z))=\frac{1}{2}$ for $|z|=1$. Hence

$$
\sum_{j=0}^{s-1}(-1)^{j} \frac{\zeta_{p}^{-l_{m} 2^{j}}}{1-\zeta_{p}^{l_{m} 2^{j}}}=\sum_{j=0}^{s-1}(-1)^{j} \zeta_{p}^{-l_{m} 2^{j}}
$$

and $S_{p, 0}^{(m)}\left(2^{a s+s / 2}\right)=S_{p, 0}^{(m)}\left(2^{a s+s / 2-1}\right)$. Since

$$
\Re\left(\frac{1}{z(1-z)\left(1-z^{2}\right)}\right)=\Re z+\frac{1}{4}-\frac{1}{4(1-\Re z)},
$$

for $|z|=1$ we obtain as above

$$
\begin{aligned}
\sum_{j=0}^{s-1}(-1)^{j} \frac{\zeta_{p}^{-l_{m} 2^{j}}}{\left(1-\zeta_{p}^{l_{m} 2^{j}}\right)\left(1-\zeta_{p}^{l_{m} 2^{j+1}}\right)} & =S_{1}-\frac{1}{4} \sum_{j=0}^{s-1}(-1)^{j} \frac{1}{1-\Re \zeta_{p}^{l_{m} 2^{j}}} \\
& =S_{1}-\frac{p^{2}}{8 \pi^{2} t} \sum_{k=1}^{2 t} \zeta_{2 t}^{k i_{m}}\left(1-(-1)^{k}\right) L\left(2, \chi_{k}\right) \\
& =S_{1}-S_{3} .
\end{aligned}
$$

Again, if

$$
\left|\frac{p^{3 / 2}}{8 \pi^{2} t} \sum_{k=1}^{2 t} \zeta_{2 t}^{i_{m}}\left(1-(-1)^{k}\right) L\left(2, \chi_{k}\right)\right|>1
$$

the above representation yields a sign change of $\psi_{p}(x)$ near $x=\frac{1}{2}$ if $\left|S_{3}\right|>\sqrt{p}$. (If $\operatorname{sgn}\left(S_{1}\right) \neq \operatorname{sgn}\left(S_{3}\right)$, then consider $S_{p, 0}^{(m)}\left(2^{a s}\left(2^{s / 2}+2^{s / 2-2}\right)\right)$.) If $p \in \mathbf{P}_{1}$ and $p \geq 17$, this concept can be used to prove a sign change of $\psi_{p}(x)$ near $x=\frac{1}{2}$ (see [2]).

However, if $t>1$ we do not know a general concept to decide whether (43) or (44) are satisfied or not. Nevertheless, it seems to be an interesting problem to consider linear combinations of values of Dirichlet $L$-series (with coefficients in a proper number field) and to quantify lower bounds in terms of $p$ and not only in terms of the heights of coefficients. We conjecture that (43) and (44) are true for sufficiently large $p \geq c(t)$.

\section{Higher PARITIES}

The purpose of this section is to show that Newman's phenomenon $S_{q, 0}(-1, n)>$ 0 (which is the same as $A_{q, 0 ; 2,0}(n)>A_{q, 0 ; 2,1}(n)$ ) has generalizations for higher parities $r>2$. However, the situation is more difficult than in the case $r=2$. We show that direct analoga of Newman's theorem appear just for $r \leq 6$ (Theorem 6). For $r>6$ we do not know whether a phenomonen of type (N1) occurs or not. But Theorem 2 has a direct analogon (Theorem 10).

Our first observation suggest that $q=2^{r}-1$ is a good choice for a phenomenon of type (N1) for a parity $r$.

Proposition 4. Let $q=2^{r}-1, r \geq 2$. Then $s(k q)=r$ for $k \leq 2^{r}$, i.e. $A_{q, 0 ; r, m}(n)=0$ for $n<2^{2 r}$ and $m \neq \equiv 0 \bmod r$.

Proof. Since $k\left(2^{r}-1\right)=(k-1) 2^{r}+\left(\left(2^{r}-1\right)-(k-1)\right)$ it is clear that $s\left(k\left(2^{r}-1\right)\right)=r$ if $k-1<2^{r}$. 
However, we will prove the following theorem, showing that (N1) holds just for $r \leq 6$

Theorem 6. The equality

$$
A_{2^{r}-1,0 ; r, 0}(n)>\max _{0<m<r} A_{2^{r}-1,0 ; r, m}(n) \quad \text { for almost all } n \geq 0
$$

holds exactly for $2 \leq r \leq 6$.

If $r>6$ it is very easy to disprove (45).

Proposition 5. Suppose that $r>6$. Then (45) fails.

Proof. We show that $\alpha_{r}>\alpha_{q, r}$. By Lemma 1 this contradicts (45).

The largest eigenvalue $\lambda_{0}\left(\zeta_{r}^{m}\right), 0<m<r$, corresponding to $\alpha_{r}$ is given by

$$
\lambda_{0}\left(\zeta_{r}\right)=\left(2 \cos \frac{\pi}{m}\right)^{r}=-2^{r}\left(1-\frac{\pi^{2}}{2 r}+\mathcal{O}\left(r^{-2}\right)\right) \text {. }
$$

Now consider any $q$-th root of unity $\zeta_{q}^{l}=e^{2 \pi x_{0} i}, 0<l<q\left(q=2^{r}-1\right)$. Then

$$
x_{0}=\frac{l}{q}=\sum_{j \geq 1} l 2^{-j r}=\sum_{k \geq 1} c_{k} 2^{-k}
$$

has a periodic digit expansion $c_{k+r}=c_{k}$, and for $\zeta_{q}^{l 2^{m}}=e^{2 \pi i x_{m}}$ we have

$$
x_{m}=\sum_{k \geq 1} c_{k+m} 2^{-k} \text {. }
$$

Furthermore there exists a $k_{0}$ with $c_{k_{0}}=1$ and $c_{k_{0}+1}=0$. Hence $1 / 2 \leq x_{k_{0}} \leq 3 / 4$, and consequently $\left|x_{k_{0}}-x_{k_{0}+1}\right| \geq 1 / 4$. Thus, for any $m$

$$
\min \left(\left|1+\zeta_{r}^{m} \zeta_{q}^{l 2^{k_{0}}} \| 1+\zeta_{r}^{m} \zeta_{q}^{l 2^{k_{0}+1}}\right|\right) \leq 2 \cos \frac{\pi}{8}
$$

which implies

$$
\left|\lambda_{l}\left(\zeta_{r}^{m}\right)\right| \leq 2^{r} \cos \frac{\pi}{8}
$$

Hence there are only finitely many $r \geq 2$ such that $\alpha_{r} \leq \alpha_{q, r}$. It is an easy task to verify that this occurs exactly for $r \leq 6$.

First, consider the case $r=3$ and set $\omega=\zeta_{3}=e^{2 \pi i / 3}$. Since

$$
S_{7,0}(\omega, n)=\sum_{m=0}^{2} A_{7,0 ; 3, m}(n) \omega^{m}
$$

(45) is equivalent to the following proposition.

Proposition 6. We have

$$
\arg \left(S_{7,0}(\omega, n)\right) \in\left(-\frac{\pi}{3}, \frac{\pi}{3}\right) \quad \text { for almost all } n \geq 0 .
$$

Proof. First, let us determine the corresponding eigenvalues $\lambda_{1}=\lambda_{\{1,2,4\}}(\omega), \lambda_{2}=$ $\lambda_{\{3,5,6\}}(\omega)$, and $\lambda_{3}=\lambda_{\{0\}}(\omega)$. Set $R=\zeta_{7}+\zeta_{7}^{2}+\zeta_{7}^{4}$ and $N=\zeta_{7}^{3}+\zeta_{7}^{5}+\zeta_{7}^{6}$. Since $R+N=-1$ and

$$
R-N=\sum_{i=1}^{6}\left(\frac{i}{7}\right) \zeta_{7}^{i}=i \sqrt{7}
$$


TABLE 6

\begin{tabular}{|c|c|c|c|c|c|c|}
\hline$j$ & $c_{j 0}^{\prime}$ & $d_{j 0}^{\prime}$ & $c_{j 1}^{\prime}$ & $d_{j 1}^{\prime}$ & $c_{j 2}^{\prime}$ & $d_{j 2}^{\prime}$ \\
\hline 0 & 18 & 0 & $18+2 \sqrt{21}$ & $-3+\sqrt{21}$ & $21+5 \sqrt{21}$ & $-3+\sqrt{21}$ \\
1 & $-3+\sqrt{21}$ & $2 \sqrt{21}$ & $-3+\sqrt{21}$ & $18+2 \sqrt{21}$ & $2 \sqrt{21}$ & $18+4 \sqrt{21}$ \\
2 & $-3+\sqrt{21}$ & $2 \sqrt{21}$ & $-3-\sqrt{21}$ & $-3+\sqrt{21}$ & $-2 \sqrt{21}$ & $18+2 \sqrt{21}$ \\
3 & $-3-\sqrt{21}$ & $-2 \sqrt{21}$ & $-3-3 \sqrt{21}$ & $-3-3 \sqrt{21}$ & $-21-5 \sqrt{21}$ & $-24-4 \sqrt{21}$ \\
4 & $-3+\sqrt{21}$ & $2 \sqrt{21}$ & $-3+3 \sqrt{21}$ & $-3+3 \sqrt{21}$ & $2 \sqrt{21}$ & $-3+\sqrt{21}$ \\
5 & $-3-\sqrt{21}$ & $-2 \sqrt{21}$ & $-3-3 \sqrt{21}$ & $-3-3 \sqrt{21}$ & 0 & $-3-3 \sqrt{21}$ \\
6 & $-3-\sqrt{21}$ & $-2 \sqrt{21}$ & $-3+\sqrt{21}$ & $-3-\sqrt{21}$ & $-2 \sqrt{21}$ & $-3-\sqrt{21}$ \\
\hline
\end{tabular}

we have $R=(-1+i \sqrt{7}) / 2$ and $N=(-1-i \sqrt{7}) / 2$. Hence

$$
\begin{aligned}
\lambda_{1} & =\left(1+\omega \zeta_{7}\right)\left(1+\omega \zeta_{7}^{2}\right)\left(1+\omega \zeta_{7}^{4}\right)=2+\omega R+\omega^{2} N \\
& =\frac{5-\sqrt{21}}{2}=0.20871 \cdots
\end{aligned}
$$

Similarly we obtain $\lambda_{2}=\left(1+\omega \zeta_{7}^{3}\right)\left(1+\omega \zeta_{7}^{5}\right)\left(1+\omega \zeta_{7}^{5}\right)=(5+\sqrt{21}) / 2=4.79128 \cdots$ and $\lambda_{3}=(1+\omega)^{3}=-1$. Thus, $\lambda_{m}=\lambda_{2}$ is the largest eigenvalue.

Next we will estimate $S_{7,0}^{(m)}(\omega, n)=c_{n 0}+\omega d_{n 0}$. Clearly it is sufficient to prove that $c_{n 0}>\left|d_{n 0}\right|$ for almost all $n \geq 0$. For this purpose we define $c_{j k}^{\prime}$ and $d_{j k}^{\prime}$ by

$$
S_{7, j}^{(m)}\left(2^{k}\right)=\frac{\lambda_{2}^{\left[\frac{k}{3}\right]}}{42}\left(c_{j k}^{\prime}+\omega d_{j k}^{\prime}\right) .
$$

Observe that $c_{j k}^{\prime}$ and $d_{j k}^{\prime}$ are periodic in $k$ with period 3 . We use (8) in order to calculate their values. First we have

$$
S_{7,0}^{(m)}\left(2^{3 l}\right)=\frac{3}{7} \lambda_{2}^{l}
$$

Next we obtain

$$
\begin{aligned}
S_{7,0}^{(m)}\left(2^{3 l+1}\right) & =\frac{\lambda_{2}^{l}}{7}\left(\left(1+\omega \zeta_{7}^{3}\right)+\left(1+\omega \zeta_{7}^{5}\right)+\left(1+\omega \zeta_{7}^{6}\right)\right)=\frac{\lambda_{2}^{l}}{7}(3+\omega N) \\
& =\frac{\lambda_{2}^{l}}{42}((18+2 \sqrt{21})+(-3+\sqrt{21}) \omega),
\end{aligned}
$$

Here and in what follows we use the representations

$$
R=\frac{(-3+\sqrt{21})+2 \sqrt{21} \omega}{6}, \quad L=\frac{(-3-\sqrt{21})-2 \sqrt{21} \omega}{6} .
$$

Similarly,

$$
\begin{aligned}
S_{7,0}^{(m)} & \left(2^{3 l+2}\right) \\
& =\frac{\lambda_{2}^{l}}{7}\left(\left(1+\omega \zeta_{7}^{3}\right)\left(1+\omega \zeta_{7}^{6}\right)+\left(1+\omega \zeta_{7}^{5}\right)\left(1+\omega \zeta_{7}^{3}\right)+\left(1+\omega \zeta_{7}^{6}\right)\left(1+\omega \zeta_{7}^{5}\right)\right) \\
& =\frac{\lambda_{2}^{l}}{7}((3-R)+(2 N-R) \omega)=\frac{\lambda_{2}^{l}}{42}((21+5 \sqrt{21})+(-3+\sqrt{21}) \omega) .
\end{aligned}
$$

The cases $j \neq 0$ can be treated in the same way. Table 6 lists the corresponding values. 
Now set $\beta=\lambda_{2}^{1 / 3}$. Then

$$
\max _{0 \leq j<7,0 \leq k<3} \beta^{k} \frac{\left|c_{j k}^{\prime}\right|+\left|d_{j k}^{\prime}\right|}{42}=\frac{3}{7} .
$$

Hence, if $n=2^{k}+\cdots$ and $S_{7, j}^{(m)}(\omega, n)=c_{n j}+\omega d_{n j}$, then

$$
\left|c_{n j}\right|+\left|d_{n j}\right| \leq \frac{3}{7} \frac{\beta^{k}}{1-\beta^{-1}}=1.0534 \ldots \beta^{k} .
$$

If $n=2^{3 l}+0 \cdot 2^{3 l-1}+\cdots$, then

$$
c_{n 0}-\left|d_{n 0}\right| \geq \frac{\beta^{3 l}}{42}\left(c_{00}^{\prime}-\left|d_{00}^{\prime}\right|\right)-1.0535 \beta^{3 l-2} \geq 0.057 \beta^{3 l} .
$$

Similarly, if $n=2^{3 l}+2^{3 l-1}+\cdots$, then

$$
\begin{aligned}
c_{n 0}-\left|d_{n 0}\right| & \geq \frac{\beta^{3 l}}{42}\left(c_{00}^{\prime}-\left|d_{00}^{\prime}\right|+\beta^{-3}\left(-d_{62}^{\prime}-\left|c_{62}^{\prime}-d_{62}^{\prime}\right|\right)\right)-1.0535 \beta^{3 l-2} \\
& \geq 0.087 \beta^{3 l}
\end{aligned}
$$

If $n=2^{3 l+1}+\cdots$, then we have to distinguish more cases. In the case $n=$ $2^{3 l+1}+0 \cdot 2^{3 l}+0 \cdot 2^{3 l-1}+\cdots$ we immediately obtain

$$
c_{n 0}-\left|d_{n 0}\right| \geq \frac{\beta^{3 l}}{42}\left(c_{01}^{\prime}-\left|d_{01}^{\prime}\right|\right)-1.0535 \beta^{3 l-2} \geq 0.23 \beta^{3 l} .
$$

If $n=2^{3 l+1}+0 \cdot 2^{3 l}+2^{3 l-1}+\cdots$, then

$c_{n 0}-\left|d_{n 0}\right| \geq \frac{\beta^{3 l}}{42}\left(c_{01}^{\prime}-\left|d_{01}^{\prime}\right|+\beta^{-3}\left(-d_{52}^{\prime}-\left|c_{52}^{\prime}-d_{52}^{\prime}\right|\right)\right)-1.0535 \beta^{3 l-2} \geq 0.23 \beta^{3 l}$.

Furthermore, if $n=2^{3 l+1}+2^{3 l}+\cdots$, then

$$
\left.c_{n 0}-\left|d_{n 0}\right| \geq \frac{\beta^{3 l}}{42}\left(c_{01}^{\prime}-\left|d_{01}^{\prime}\right|-d_{50}^{\prime}-\left|c_{50}^{\prime}-d_{50}^{\prime}\right|\right)\right)-1.0535 \beta^{3 l-1} \geq 0.16 \beta^{3 l} .
$$

Finally, the case $n=2^{3 l+2}+\cdots$ can be treated in the same way. Hence

$$
c_{n 0}-\left|d_{n 0}\right| \geq c \lambda_{2}^{(\log n) /(3 \log 2)},
$$

and consequently (46).

Similarly to the first part of Theorem 1, we are also able to provide infinitely many examples for phenomena of type (N1) for parity $r=3$.

Theorem 7. Suppose that $r=3$ and that $q$ is an odd multiple of 7 . Then (N1) and (N2) hold.

The essential part of the proof is to identify the largest eigenvalue. This will be done in the following lemma.

Lemma 10. Suppose that $q$ is a positive odd integer. Then any eigenvalue

$$
\lambda_{l}(\omega)=\prod_{m=0}^{s-1}\left(1+\omega \zeta_{q}^{l 2^{m}}\right)
$$

of $\mathbf{M}(\omega)$ is bounded by $\left|\lambda_{l}(\omega)\right|<((5+\sqrt{21}) / 2)^{s / 3}$ or $\lambda_{l}(\omega)=((5+\sqrt{21}) / 2)^{s / 3}$.

The case $\lambda_{l}(\omega)=((5+\sqrt{21}) / 2)^{s / 3}$ appears if and only if $q \equiv 0 \bmod 7$ and either $l \equiv 3 q / 7 \bmod q$ or $l \equiv 5 q / 7 \bmod q$ or $l \equiv 6 q / 7 \bmod q$. 
Proof. Let $\lambda_{l}(\omega)=\prod_{m=0}^{s-1}\left(1+\omega \zeta_{q}^{l 2^{m}}\right)$ be an eigenvalue of $\mathbf{M}(\omega)$. If $l \equiv 3 q / 7 \bmod q$ or $l \equiv 5 q / 7 \bmod q$ or $l \equiv 6 q / 7 \bmod q$, then $\lambda_{l}(\omega)=((5+\sqrt{21}) / 2)^{s / 3}$.

In the remaining cases we use the following partition: $M_{1}, M_{2}=M_{1}+1, M_{3}=$ $M_{1}+2, M_{4}, M_{5}=M_{4}+1, M_{6}=M_{4}-1, M_{7}$ of $\{0,1, \ldots, s-1\} . M_{1}$ consists of those $m$ such that $\arg \left(\zeta_{q}^{l 2^{m}}\right) \in(-4 \pi / 7,-2 \pi / 7)$ and $M_{4}$ of those $m$ which are not contained in $M_{2}$ and satisfy $\arg \left(\zeta_{q}^{l 2^{m}}\right) \in(-8 \pi / 7,-4 \pi / 7)$. Set

$$
\begin{aligned}
& f(x)=8\left|\cos \left(\frac{x}{2}+\frac{\pi}{3}\right) \cos \left(x+\frac{\pi}{3}\right) \cos \left(2 x+\frac{\pi}{3}\right)\right|, \\
& g(x)=8\left|\cos \left(\frac{x}{2}+\frac{\pi}{3}\right) \cos \left(x+\frac{\pi}{3}\right) \cos \left(\frac{x}{4}-\frac{\pi}{3}\right)\right| .
\end{aligned}
$$

Then $f(-2 \pi / 7)=(5+\sqrt{21}) / 2$ and

$$
f(x)=\left|\left(1+\omega e^{i x}\right)\left(1+\omega e^{2 i x}\right)\left(1+\omega e^{4 i x}\right)\right|<f(-2 \pi / 7)
$$

for $x \in(-4 \pi / 7,-2 \pi / 7)$. Hence

$$
\prod_{m \in M_{1} \cup M_{2} \cup M_{3}}\left|1+\omega \zeta_{q}^{l^{2}}\right|<\left(\frac{5+\sqrt{21}}{2}\right)^{\left|M_{1}\right|} .
$$

Similarly, $g(x)<f(-2 \pi / 7), x \in(-8 \pi / 7,-4 \pi / 7)$, implies

$$
\prod_{m \in M_{4} \cup M_{5} \cup M_{6}}\left|1+\omega \zeta_{q}^{l 2^{m}}\right|<\left(\frac{5+\sqrt{21}}{2}\right)^{\left|M_{4}\right|} .
$$

Finally, $\left|1+\omega e^{i x}\right|<f(-2 \pi / 7)^{1 / 3}, x \in(-4 \pi / 7,6 \pi / 7)$, provides

$$
\left|1+\omega \zeta_{q}^{l 2^{m}}\right|<\left(\frac{5+\sqrt{21}}{2}\right)^{1 / 3}
$$

for all $m \in M_{7}$, which completes the proof of Lemma 10 .

Now the proof of Theorem 7 is almost the same as the proof of Proposition 6 . Therefore we will not give the details here.

Next, let $r=4$. Here we prove.

Proposition 7. We have

$$
\arg \left(S_{15,0}(i, n)\right) \in\left(-\frac{\pi}{4}, \frac{\pi}{4}\right) \quad \text { for almost all } n \geq 0 \text {. }
$$

It is easy to verfy that Proposition 7 implies Theorem 6 for $r=4$. Since (47) is equivalent to

$$
A_{15,0 ; 4,0}(n)-A_{15,0 ; 4,2}(n)>\left|A_{15,0 ; 4,1}(n)-A_{15,0 ; 4,3}(n)\right|,
$$

we have $A_{15,0 ; 4,0}(n)>A_{15,0 ; 4,2}(n)$. By Theorem $1(q=15)$ we also know that

$$
A_{15,0 ; 4,0}(n)+A_{15,0 ; 4,2}(n)>A_{15,0 ; 4,1}(n)+A_{15,0 ; 4,3}(n) .
$$

Let $\{k, l\}=\{1,3\}$ and suppose that $A_{15,0 ; 4, k}(n) \geq A_{15,0 ; 4, l}(n)$. Then (48) and (49) imply

$$
A_{15,0 ; 4,0}(n)>A_{15,0 ; 4, k}(n) \geq A_{15,0 ; 4, l}(n),
$$

and consequently (45). 
TABLE 7.1

\begin{tabular}{|r|c|c|c|c|}
\hline$j$ & $c_{j 0}^{\prime}$ & $d_{j 0}^{\prime}$ & $c_{j 1}^{\prime}$ & $d_{j 1}^{\prime}$ \\
\hline 0 & 8 & 0 & $8+\sqrt{15}$ & 1 \\
1 & 1 & $\sqrt{15}$ & 1 & $8+\sqrt{15}$ \\
2 & 1 & $\sqrt{15}$ & $1-\sqrt{15}$ & $1+\sqrt{15}$ \\
3 & -2 & 0 & $-2-\sqrt{15}$ & 1 \\
4 & 1 & $\sqrt{15}$ & 1 & $-2+\sqrt{15}$ \\
5 & -4 & 0 & $4-\sqrt{15}$ & 1 \\
6 & -2 & 0 & -2 & -4 \\
7 & 1 & $-\sqrt{15}$ & 1 & $-2-\sqrt{15}$ \\
8 & 1 & $\sqrt{15}$ & $1+\sqrt{15}$ & $1+\sqrt{15}$ \\
9 & -2 & 0 & $-2-\sqrt{15}$ & 1 \\
10 & -4 & 0 & -4 & -2 \\
11 & 1 & $-\sqrt{15}$ & 1 & $-4-\sqrt{15}$ \\
12 & -2 & 0 & $-2+\sqrt{15}$ & 1 \\
13 & 1 & $-\sqrt{15}$ & 1 & $-2-\sqrt{15}$ \\
14 & 1 & $-\sqrt{15}$ & $1+\sqrt{15}$ & $1-\sqrt{15}$ \\
\hline
\end{tabular}

It should also be mentioned that $\Re\left(S_{15,0}(i, n)\right)>0$ for almost all $n$ is also sufficient to prove (45). By (6) we have

$$
A_{15,0 ; 4, m}(n)=\frac{1}{4} \sum_{l=0}^{3} i^{-l m} S_{15,0}\left(i^{l}, n\right) .
$$

Hence $\Re\left(S_{15,0}(i, n)\right)>0$ implies $A_{15,0 ; 4,0}(n)>A_{15,0 ; 4,2}(n)$. Furthermore, by Theorem $1 S_{15,0}(-1, n) \gg n^{\frac{\log 3}{\log 4}}$. Consequently we also have

$$
A_{15,0 ; 4,0}(n)>\max \left(A_{15,0 ; 4,1}(n), A_{15,0 ; 4,3}(n)\right)
$$

for sufficienty large $n$.

Proof of Proposition 7. The computation of the eigenvalues of $\mathbf{M}(i)$ can be worked out explicitly:

$$
\begin{aligned}
\lambda_{1} & =\lambda_{\{1,2,4,8\}}=\left(1+i \zeta_{1} 5\right)\left(1+i \zeta_{1} 5^{2}\right)\left(1+i \zeta_{1} 5^{4}\right)\left(1+i \zeta_{1} 5^{8}\right) \\
& =2-\left(\zeta_{1} 5^{3}+\zeta_{1} 5^{6}+\zeta_{1} 5^{9}+\zeta_{1} 5^{12}\right)-\left(\zeta_{1} 5^{5}+\zeta_{1} 5^{10}\right)+i \sum_{j=1}^{14}\left(\frac{j}{15}\right) \zeta_{1} 5^{j} \\
& =4-\sqrt{15}
\end{aligned}
$$

where $\left(\frac{\dot{15}}{15}\right)$ denotes the Jacobi-Kronecker symbol. The other eigenvalues are given by $\lambda_{2}=\lambda_{\{14,7,11,13\}}=4+\sqrt{15}, \lambda_{3}=\lambda_{\{3,6,9,12\}}=1, \lambda_{4}=\lambda_{\{5,10\}}=-1$, and by $\lambda_{5}=\lambda_{\{0\}}=-4$. Hence the largest eigenvalue is $\lambda_{2}$. Now we can proceed as in the proof of Proposition 6. We just reproduce a table (Tables 7.1 and 7.2) for $c_{j k}^{\prime}$ and $d_{j k}^{\prime}$ defined by

$$
S_{15, j}^{(m)}\left(i, 2^{k}\right)=\frac{\lambda_{2}^{\left[\frac{k}{4}\right]}}{30}\left(c_{j k}^{\prime}+i d_{j k}^{\prime}\right)
$$




\begin{tabular}{|r|c|c|c|c|}
\hline$j$ & $c_{j 2}^{\prime}$ & $d_{j 2}^{\prime}$ & $c_{j 3}^{\prime}$ & $d_{j 3}^{\prime}$ \\
\hline 0 & $10+2 \sqrt{15}$ & 2 & $16+4 \sqrt{15}$ & 2 \\
1 & $\sqrt{15}$ & $9+2 \sqrt{15}$ & $3+\sqrt{15}$ & $9+3 \sqrt{15}$ \\
2 & $-\sqrt{15}$ & $9+2 \sqrt{15}$ & 1 & $14+3 \sqrt{15}$ \\
3 & $-10-2 \sqrt{15}$ & 2 & $-9-2 \sqrt{15}$ & $2+\sqrt{15}$ \\
4 & $-\sqrt{15}$ & -1 & $-2-\sqrt{15}$ & $9+2 \sqrt{15}$ \\
5 & $-9-\sqrt{15}$ & $-1-\sqrt{15}$ & $-14-\sqrt{15}$ & $-1+2 \sqrt{15}$ \\
6 & $-\sqrt{15}$ & -3 & $-9-\sqrt{15}$ & $-3-3 \sqrt{15}$ \\
7 & 0 & $-6-2 \sqrt{15}$ & -2 & $-16-4 \sqrt{15}$ \\
8 & $5+\sqrt{15}$ & $-1+\sqrt{15}$ & $6+\sqrt{15}$ & -1 \\
9 & 0 & 2 & $1+\sqrt{15}$ & $-3-\sqrt{15}$ \\
10 & $-5-\sqrt{15}$ & $-1-\sqrt{15}$ & $-2-\sqrt{15}$ & -1 \\
11 & 0 & $-6-2 \sqrt{15}$ & $6+2 \sqrt{15}$ & $-6-2 \sqrt{15}$ \\
12 & $\sqrt{15}$ & -3 & 1 & $2+\sqrt{15}$ \\
13 & $5+\sqrt{15}$ & $-1-\sqrt{15}$ & $3+\sqrt{15}$ & $-1-\sqrt{15}$ \\
14 & $\sqrt{15}$ & -1 & 1 & $-6-\sqrt{15}$ \\
\hline
\end{tabular}

Finally, let us consider the cases $r=5$ and $r=6$. In the case $r=5$ it suffices to show that

$$
\Re\left(S_{31,0}\left(\zeta_{5}, n\right)\right)>\Re\left(\zeta_{5}^{-m} S_{31,0}\left(\zeta_{5}, n\right)\right) \quad(m \neq \equiv 0 \bmod 5),
$$

which can be checked by considering the largest eigenvalue $\lambda_{-1}\left(\zeta_{5}\right)$ and similar calculations as above. (Again a simple computer program assists us.)

The case $r=6$ is interesting because (45) can be deduced from Theorems 1 and 7. By (6)

$$
A_{63,0 ; 6, m}(n)=\frac{1}{6} \sum_{l=0}^{5} \zeta_{6}^{-l m} S_{63,0}\left(\zeta_{6}^{l}, n\right) .
$$

By Theorem $7, \arg \left(S_{63,0}\left(\zeta_{6}^{2}, n\right)\right) \in(-\pi / 3, \pi / 3)$. Thus, for sufficiently large $n$,

$$
A_{63 ; 0 ; 6,0}(n)>\max \left(A_{63 ; 0 ; 6,2}(n), A_{63 ; 0 ; 6,4}(n)\right),
$$

since the largest eigenvalue of $\mathbf{M}\left(\zeta_{6}^{2}\right)$ is larger than the largest eigenvalue of $\mathbf{M}\left(\zeta_{6}\right)$. Furthermore, by Theorem $1 S_{63,0}(-1, n) \gg n^{\frac{\log 3}{\log 4}}$, and consequently

$$
A_{63 ; 0 ; 6,0}(n)>\max \left(A_{63 ; 0 ; 6,1}(n), A_{63 ; 0 ; 6,3}(n), A_{63 ; 0 ; 6,5}(n)\right)
$$

for sufficientely large $n$.

Therefore we have provided a complete answer for the case $q=2^{r}-1$ with respect to $(\mathbf{N 1})$. However, the situation is much more delicate when we consider (N2) instead of (N1).

Theorem 8. We have

$$
R_{127,0 ; 7,0}(n)>0 \text { for almost all } n \geq 0 .
$$

This means that (N2) holds for $r=7$ although (N1) fails. (We do not give a detailed proof. We only want to mention that it suffices to show that $\Re\left(S_{127,0}\left(\zeta_{7}, n\right)\right)$ $>0$.) Therefore it might be possible that (N2) holds for all $r \geq 2$. But again the answer is negative. 
Theorem 9. There are infinitely many $r \geq 2$ such that

$$
R_{2^{r}-1,0 ; r, 0}(n)<0 \quad \text { for infinitely many } n \geq 0 .
$$

Sketch of the Proof. It is sufficient to show that there are infinitely many $r \geq 2$ such that the eigenvalue $\lambda_{l}\left(\zeta_{r}^{m}\right), 0<l<2^{r}-1,0<m<r$, of largest modulus $\left|\lambda_{m}\right|$ is negative. In what follows we will indicate that if there exists a positive integer $m_{r}$ such that $\left|\sqrt{r} / \pi+C-m_{r}\right|<1 / 4$ (where $C$ a real constant and $r \geq r_{0}$ is sufficiently large), then $\lambda_{1}\left(\zeta_{r}^{-m_{r}}\right)$ is the eigenvalue of largest modulus

$$
\left|\lambda_{m}\right|=\left|\lambda_{1}\left(\zeta_{r}^{-m_{r}}\right)\right| \sim \frac{2^{r} e^{-1 / 2}}{2 \pi \sqrt{r}}
$$

Since

$$
\arg \left(\lambda_{1}\left(\zeta_{r}^{-m_{r}}\right)\right)=\sum_{j=0}^{r-1} \pi\left(\frac{2^{j}}{2^{r}-1}-\frac{m_{r}}{r}\right)=\pi\left(1-m_{r}\right),
$$

we have $\operatorname{sgn}\left(\lambda_{1}\left(\zeta_{r}^{-m_{r}}\right)\right)<0$ if $m_{r}$ is even. Obviously, this case occurs infinitely many times.

We use the fact that the digit expansion of $x_{0}=l /\left(2^{r}-1\right)=\sum_{k \geq 1} c_{k} 2^{-k}$ is periodic, i.e. $c_{k+r}=c_{k}$, and that $x_{j}=\sum_{k \geq 1} c_{k+j} 2^{-k}$ satisfies $\zeta_{2^{r}-1}^{l 2^{j}}=e^{2 \pi i x_{j}}$. (Compare with the proof of Proposition 5.) By considering several subcases it turns out that if $l$ is unbounded, then

$$
\left|\lambda_{l}\left(\zeta_{r}^{m}\right)\right|=o\left(2^{r} r^{-1 / 2}\right)
$$

Conversely, if $l$ is bounded, then

$$
\max _{m}\left|\lambda_{l}\left(\zeta_{r}^{m}\right)\right| \sim \frac{2^{r-1} e^{-1 / 2}}{l \pi \sqrt{r}}
$$

in which the maximum is attained for $|m| \sim \sqrt{r} / \pi$. Therefore $l=1,|m| \sim \sqrt{r} / \pi$ is the only relevant case. (Since $\left|1+\zeta_{r}^{m} \zeta_{q}^{2^{j}}\right|<\left|1+\zeta_{r}^{-m} \zeta_{q}^{2^{j}}\right|(m>0)$, we may also assume that $m \sim-\sqrt{r} / \pi$.) A more detailed analysis shows that the maximum value of $\left|\lambda_{1}\left(\zeta_{q}^{m}\right)\right|$ is attained for

$$
m=-\frac{\sqrt{r}}{\pi}-C+\mathcal{O}\left(r^{-1 / 2}\right),
$$

in which $m$ is assumed to be a continuous real parameter and $C$ is a computable constant. Furthermore, if $\sqrt{r} / \pi+C$ is near to an integer $m_{r}$, e.g. $\left|\sqrt{r} / \pi+C-m_{r}\right|<$ $1 / 4$, and if $r$ is sufficiently large, then $\left|\lambda_{m}\right|=\left|\lambda_{1}\left(\zeta_{q}^{-m_{r}}\right)\right|$.

We finish this section on higher parities with an analogue to Theorem 2 .

Theorem 10. For any $r>1$ there exists a constant $C_{r}>0$ such that for any $t \geq 1$ primes $q \in \mathbf{P}_{t}$ satisfying $(\mathbf{N 1})$ or $(\mathbf{N 2})$ are bounded by

$$
q \leq C_{r} t^{4} \log ^{4} t .
$$

For the proof we can use a similar procedure as above. Instead of (40) we need the following formula. 
Proposition 8. Suppose that $p$ is an odd prime and $s=\operatorname{ord}_{p}(2)$. If $y \in \mathbf{C}$ has modulus $|y|=1$, then for any $\mathbf{l} \in L$

$$
\begin{aligned}
& \Re\left(\sum_{l \in \mathbf{l}} \frac{1}{\left(1+y \zeta_{p}^{l}\right)\left(1+y \zeta_{p}^{2 l}\right)}\right) \\
& \quad=\frac{s}{2}-\frac{1}{4} \sum_{l \in \mathbf{l}} \frac{1}{1-\Re \zeta_{p}^{2 l}}\left(1+2 \frac{\cos (\arg y / 2)}{\cos \left((\arg y) / 2+\arg \zeta_{p}^{l}\right)}\right) .
\end{aligned}
$$

Proof. From

$$
\begin{aligned}
s= & \sum_{l \in \mathbf{1}} \frac{1+y \zeta_{p}^{l}+y \zeta^{2 l}+y^{2} \zeta^{3 l}}{\left(1+y \zeta_{p}^{l}\right)\left(1+y \zeta_{p}^{2 l}\right)} \\
= & \sum_{l \in \mathbf{l}} \frac{1}{\left(1+y \zeta_{p}^{l}\right)\left(1+y \zeta_{p}^{2 l}\right)}+\sum_{l \in \mathbf{l}} \frac{1}{\left(1+\bar{y} \zeta_{p}^{-l}\right)\left(1+\bar{y} \zeta_{p}^{-2 l}\right)} \\
& +\sum_{l \in \mathbf{l}} \frac{y \zeta_{p}^{l}\left(1+\zeta_{p}^{l}\right.}{\left(1+y \zeta_{p}^{l}\right)\left(1+y \zeta_{p}^{2 l}\right)}
\end{aligned}
$$

we obtain

$$
\Re\left(\sum_{l \in \mathbf{1}} \frac{1}{\left(1+y \zeta_{p}^{l}\right)\left(1+y \zeta_{p}^{2 l}\right)}\right)=\frac{s}{2}-\frac{1}{2} \sum_{l \in \mathbf{l}} \frac{y \zeta_{p}^{l}\left(1+\zeta_{p}^{l}\right.}{\left(1+y \zeta_{p}^{l}\right)\left(1+y \zeta_{p}^{2 l}\right)}=\frac{s}{2}-\frac{1}{2} S(y),
$$

where the mapping $y \mapsto S(y), y \neq-\zeta_{p}^{-l}$, is continuous. In particular,

$$
S(-1)=-\sum_{l \in \mathbf{l}} \frac{\zeta_{p}^{l}}{\left(1-\zeta_{p}^{l}\right)^{2}}=\frac{1}{2} \sum_{l \in \mathbf{l}} \frac{1}{1-\Re \zeta_{p}^{l}}
$$

By using a partial fraction expansion it follows that $S(y), y \neq 1$, can be represented by

$$
\begin{aligned}
S(y) & =\frac{1-y}{1+y} \sum_{l \in \mathbf{1}} \frac{1}{\left(1+y \zeta_{p}^{l}\right)}-\frac{1-y}{1+y} \sum_{l \in \mathbf{l}} \frac{1}{1+y \zeta_{p}^{2 l}}+\frac{2 y}{1+y} \sum_{l \in \mathbf{l}} \frac{\zeta_{p}^{l}}{1+y \zeta_{p}^{2 l}} \\
& =\frac{2 y}{1+y} \sum_{l \in \mathbf{l}} \frac{1}{\zeta_{p}^{-l}+y \zeta_{p}^{l}} .
\end{aligned}
$$

Since $S(-1)$ is finite, it follows that

$$
\sum_{l \in \mathbf{l}} \frac{1}{\zeta_{p}^{-l}-\zeta_{p}^{l}}=0
$$


and consequently

$$
\begin{aligned}
S(y) & =\frac{2 y}{1+y} \sum_{l \in \mathbf{l}}\left(\frac{1}{\zeta_{p}^{-l}+y \zeta_{p}^{l}}-\frac{1}{\zeta_{p}^{-l}-\zeta_{p}^{l}}\right) \\
& =-\Re\left(\sum_{l \in \mathbf{l}} \frac{y \zeta_{p}^{l}}{\left(\zeta_{p}^{-l}+y \zeta_{p}^{l}\right)\left(\zeta_{p}^{-l}-\zeta_{p}^{l}\right)}\right) \\
& =-\sum_{l \in \mathbf{l}} \frac{\zeta_{p}^{-l}-y \zeta_{p}^{l}}{\left(\zeta_{p}^{-l}+y \zeta_{p}^{l}\right)\left(\zeta_{p}^{-l}-\zeta_{p}^{l}\right)} \\
& =S(-1)-\sum_{l \in \mathbf{l}}\left(\frac{\zeta_{p}^{-l}-y \zeta_{p}^{l}}{\left(\zeta_{p}^{-l}+y \zeta_{p}^{l}\right)\left(\zeta_{p}^{-l}-\zeta_{p}^{l}\right)}-\frac{\zeta_{p}^{-l}+\zeta_{p}^{l}}{\left(\zeta_{p}^{-l}-\zeta_{p}^{l}\right)^{2}}\right) \\
& =S(-1)-\sum_{l \in \mathbf{l}} \frac{1+y}{\left(\zeta_{p}^{-l}-\zeta_{p}^{l}\right)^{2}\left(\zeta_{p}^{-l}+y \zeta_{p}^{l}\right)} \\
& =\frac{1}{2} \sum_{l \in \mathbf{l}} \frac{1}{1-\Re \zeta_{p}^{l}}+\sum_{l \in \mathbf{l}} \frac{1}{1-\Re \zeta_{p}^{2 l}} \frac{1+y}{\zeta_{p}^{-l}+y \zeta_{p}^{l}}
\end{aligned}
$$

which proves $(50)$.

The essential difference between the proofs of Theorem 2 and Theorem 10 is that you have to take into account the sign of

$$
\frac{\cos (m \pi / r)}{\cos \left(m \pi / r+\arg \zeta_{p}^{l}\right)} \text {. }
$$

Let $\mathbf{1}^{-}$denote the set of those $l \in \mathbf{l}$ such that this sign is negative. Then it is an easy exercise to show that

$$
\sum_{l \in \mathbf{1}^{-}} \frac{1}{1-\Re \zeta_{p}^{2 l}} \frac{\cos (m \pi / r)}{\cos \left(m \pi / r+\arg \zeta_{l}\right)}=\mathcal{O}_{r}(p \log p) .
$$

You only have to verify that $1-\Re \zeta_{p}^{2 l}>c_{r}$ for $l \in \mathbf{1}^{-}$and that $\arg \zeta_{l}$ is different for different $l \in \mathrm{l}$. Hence, if $p>C_{r}(t \log p)^{4}$ (for a sufficiently large constant $C_{r}>0$ ), then

$$
\frac{1}{8 \pi 1^{2}} \frac{p^{3 / 2}}{t^{2} \log p}>\frac{p}{2 t}+\mathcal{O}_{r}(p \log p),
$$

which implies that $\Re\left(S_{p, 0}\left(\zeta_{r}^{m}, 2^{2 a s-2}\right)\right)<0$ for sufficiently large $a$.

\section{REFERENCES}

[1] J. Coquet, A summation formula related to the binary digits, Invent. Math. 73 (1983), 107115. MR 85c: 11012

[2] M. Drmota and M. Skałba, Sign-changes of the Thue-Morse sequence and Dirichlet L-series, manuscripta math. 86 (1995), 519-541. MR 96b:11027

[3] J. M. Dumont, Discrépance des progressions arithméthiques dans la suite de Morse, C. R. Acad. Sci. Paris, Série I 297 (1983), 145-148. MR 85f:11058

[4] P. Erdős, Bemerkungen zu einer Aufgabe von Elementen, Archiv Math. 27 (1976), 159-163. MR 53:7969

[5] S. Goldstein, K. A. Kelly, and E. R. Speer, The fractal structure of rarefied sums of the Thue-Morse sequence, J. Number Th. 42 (1992), 1-19. MR 93m:11020

[6] P. J. Grabner, A note on the parity of the sum-of-digits function, Actes $30^{\mathrm{e}}$ Séminaire Lotharingien de Combinatoire, 1993, 35-42. MR 95k:11125 
[7] P. J. Grabner, T. Herendi, and R. F. Tichy, Fractal digital sums and codes, Appl. Algebra Engin. Comm. Comput. 1 (1997), 33-39. CMP 97:17

[8] H. Leinfellner, Thesis, TU Wien, 1998.

[9] M. Morse, Reccurent geodesics on a surface of negative curvature, Trans. Amer. Math. Soc. 22 (1921), 84-100.

[10] D. J. Newman, On the number of binary digits in a multiple of three, Proc. Am. Math. Soc. 21 (1969), 719-721. MR 39:5466

[11] K. Prachar, 'Primzahlverteilung,' Springer, Berlin, 1957. MR 19:393b

[12] I. M. Vinogradov, 'Elemente der Zahlentheorie,' Oldenbourg, München, 1956.

Department of Geometry, Technical University of Vienna, Wiedner Hauptstrasse 8-10, A-1040 Vienna, Austria

E-mail address: michael.drmota@tuwien.ac.at

Institute of Mathematics, Warsaw University, Banacha 2, 02-097 Warsaw, Poland

E-mail address: skalba@mimuw.edu.pl 